\title{
Silicon Photonic Integration Platform- Have We Found the Sweet Spot?
}

\author{
Dan-Xia Xu, Jens H. Schmid, Graham T. Reed, Goran Z. Mashanovich, David J. Thomson, Milos Nedeljkovic, \\ Xia Chen, Dries Van Thourhout, Shahram Keyvaninia, and Shankar K. Selvaraja
}

(Invited Paper)

\begin{abstract}
The current trend in silicon photonics towards higher levels of integration as well as the model of using CMOS foundries for fabrication are leading to a need for standardization of substrate parameters and fabrication processes. In particular, for several established research and development foundries that grant general access, silicon-on-insulator wafers with a silicon thickness of $220 \mathrm{~nm}$ have become the standard substrate for which devices and circuits have to be designed. In this study we investigate the role of silicon device layer thickness in design optimization of various components that need to be integrated in a typical optical transceiver, including both passive ones for routing, wavelength selection, and light coupling as well as active ones such as monolithic modulators and on-chip lasers produced by hybrid integration. We find that in all devices considered there is an advantage in using a silicon thickness larger than $220 \mathrm{~nm}$, either for improved performance or for simplified fabrication processes and relaxed tolerances.
\end{abstract}

Index Terms-Integrated optics, silicon photonics, photonic integration, optical interconnect, optoelectronics.

\section{INTRODUCTION}

$\mathbf{F}$ ROM long distance telecommunication to intra-chip computer interconnects, the demand for data transport bandwidth continues to grow. To overcome the limitations of traditional copper-based technologies, optical solutions have penetrated ever deeper into the communications fabric [1], [2]. Further deployment of optical solutions requires that the new technologies satisfy similar demands facing the microelectronics industry: increased power efficiency, increased performance

Manuscript received October 17, 2013; revised December 23, 2013; accepted December 31, 2013. Date of publication January 10, 2014; date of current version February 7, 2014.

D.-X. Xu and J. H. Schmid are with Information and Communication Technologies, National Research Council Canada, Ottawa, ON K1 A 0R6, Canada (e-mail: Danxia.xu@nrc-cnrc.gc.ca; jens.schmid@nrc-cnrc.gc.ca).

G. T. Reed, G. Z. Mashanovich, D. J. Thomson, M. Nedeljkovic, and X. Chen are with the Optoelectronics Research Centre, University of Southampton, Southampton, SO17 1BJ, U.K. (e-mail: g.reed@soton.ac.uk; g.mashanovich@ soton.ac.uk; D.Thomson@soton.ac.uk; m.nedeljkovic@soton.ac.uk; xc1y11@ ecs.soton.ac.uk).

D. Van Thourhout and S. Keyvaninia are with the Photonics Research Group, Department of Information Technology, Ghent University imec, B-9000 Ghent, Belgium (e-mail: dries.vanthourhout@intec.ugent.be; shahram.keyvaninia@intec.ugent.be).

S. K. Selvaraja is with imec, B-3000 Leuven, Belgium (e-mail: selvara@ imec.be).

Color versions of one or more of the figures in this paper are available online at http://ieeexplore.ieee.org.

Digital Object Identifier 10.1109/JSTQE.2014.2299634 and reduced cost. To that end, silicon photonics has gained increasing acceptance as the platform of choice for photonic integration to meet the requirements in optical communications, with all components built on silicon-on-insulator (SOI) substrates through monolithic or hybrid integration [2]-[8]. Through the research of the last two decades, the performance of many discrete silicon photonic components has been improved to match or even out-perform that of their traditional counterparts [9]-[12]. In recent years, more effort has been directed towards the integration of multiple functions into photonic integrated circuits (PICs) on chip to realize the true advantage of silicon photonics [7], [13]-[16]. During the phase of optimizing individual components, the silicon waveguide structures have been chosen to meet the respective needs in each situation. For passive devices, modulators and photodetectors, the thickness of the silicon device layer typically ranges from $200 \mathrm{~nm}$ to $400 \mathrm{~nm}$ [9], [17]-[26]. The hybrid integration of III-V lasers using bonding techniques has been demonstrated by several groups [12], [27], but most are on SOI structures with a silicon thickness of at least $400 \mathrm{~nm}$ in order to achieve efficient evanescent coupling between the laser and the silicon waveguide [29]. Since large scale integration is the ultimate goal of silicon photonics, a common platform that optimizes all considerations is the necessary starting point. Although waveguide widths can be easily modified across a photonic circuit, modifying the silicon thickness locally requires additional fabrication steps and often high precision alignments. The silicon thickness of the SOI substrates should therefore be carefully chosen by weighing the trade-offs between various factors.

Another important trend in silicon photonics is the emergence of fabrication foundries [30]-[33]. Due to the high cost associated with the fabrication of sophisticated state-of-the-art devices, many research labs and companies have adopted the fabless model and use speciality CMOS foundries that are publicly accessible to prototype their own designs. Cost saving is particularly significant for multi-project wafer (MPW) runs, allowing new players to enter the silicon photonics field at a modest expense, and accelerated the pace of research and development (R\&D). Initial attempts of offering flexible choices for the silicon thickness in the starting SOI wafers have proven too costly. Now established silicon photonics foundries around the world, namely EpixFab (IMEC and LETI) and A*Star/IME, have converged to using SOI wafers with $220 \mathrm{~nm}$ thick silicon for their MPW runs [33]. Passive components, plasma dispersion modulators and Ge photodetectors are all offered on this platform. 

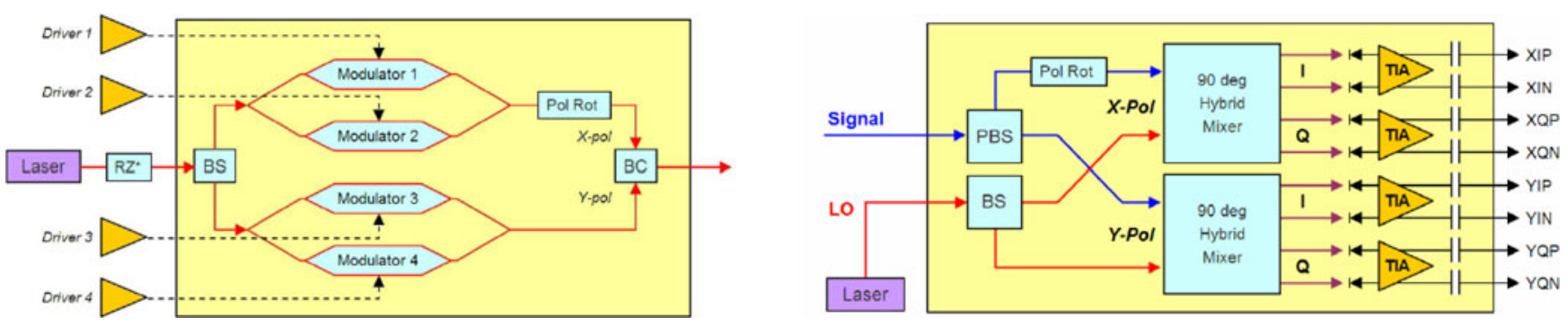

Fig. 1. Coherent transceiver modules. Left: Block diagram of a DP-QPSK transmitter module; Right: Block diagram of a DP-QPSK receiver module, shown with balanced detection and outputs. Source: www.oiforum.com (Optical internetworking forum).

Consequently, this particular choice has become the de facto platform of photonic integration for much of the research community and small companies. Commercially available optical transceiver cables from Luxtera/Molex are based on SOI with 300-310 nm thick silicon, which was chosen to accommodate low loss compact passive devices and for a bulk-like transistor process [34], [35]. Silicon photonics development at Oracle is also based on this platform [36]. Even though the foundry service on this platform has been made available since 2011, it has not been widely adopted by the research community. Other strategies to deal with some of the conflicting requirements in integration are being explored, while the trend of 'standardization' is moving ahead. Economic analysis suggests that the incentive for using silicon photonics, i.e., the existing capital-intensive infrastructure in Si-CMOS, can also become its own demon. In CMOS manufacturing, competitiveness relies on high product volumes and manufacturing yield. At this juncture, we ask these questions: How can the requirements from key building blocks best be met with satisfactory trade-offs? Is there a 'sweet spot' in the silicon layer thickness that enables optimizing the performance of devices, minimizing fabrication complexities, and maximizing tolerances to the inevitable variations in fabrication? Are there important remaining issues? Have we, the silicon photonics community, found the 'sweet spot' for silicon photonic integration?

In this paper, we examine the optimization considerations for passive and active components required in an optical transceiver for fiber links as a case study, since it is currently the main driver for silicon PICs. Requirements for chip-level computer interconnects share many commonalities, although it is sufficient to use one polarization since silicon waveguides are polarization maintaining. Our analysis is focused on the integration scenario of using monolithic silicon carrier-depletion modulators and hybrid lasers, a strategy seen as the path for near to medium term commercialization. State-of-the-art development of key building blocks is reviewed. We highlight their common as well as sometimes conflicting requirements on the silicon waveguide structures and the operating polarizations. In our discussions, we not only consider the device performance, but also pay particular attention to the fabrication tolerance. Research that emerged recently to address some of these concerns is reviewed. Potential alternative strategies to meet the diverging requirements in photonic integration are explored, with the intention of inviting debate and further exploration from the research community. We believe continued research in photonic devices over a range of dimension and material options is still vital to achieve farreaching commercialization of silicon photonics and to provide guidance for evolving standardization.

\section{OVERVIEW OF KEY COMPONENTS IN AN OPTICAL TRANSCEIVER AND KEY CHALLENGES}

For data transport at all distances, the key components include filters/routers, lasers, modulators and photodetectors. Fig. 1 illustrate the block diagram of a coherent optical transceiver with dual-polarization and quadrature phase-shift keying (DP-QPSK), as defined by the Optical Internetworking Forum, mainly considered for long-haul communications at present. A simple method for increasing data capacity is to split the input light into multiple channels and then encode each stream separately [37]. Multiple fibers are then used to capture the output signals. The wavelength-division-multiplexing technique is often used to further increase the data capacity by combining signals carried on different wavelengths in a single transmission fiber, using multiplexers and demultiplexers. In the transmitter section, only one polarization is used for traditional on-offkeying signals, while for dual-polarization keying, the addition of polarization rotators (PR) and a polarization beam splitters (PBS, which also can serve as polarization combiners) right before coupling to the output fiber suffice to complete the function. For applications with a high data rate and a transmission distance of more than 1 kilometer, single mode edge emitting lasers are generally used. Since edge-emitting lasers launch light with transverse electric (TE) field, this polarization is the natural choice for the subsequent components such as the demultiplexers and modulators. In the receiver section, signals in all polarizations need to be recovered since the currently installed optical fibers do not maintain the orientation of the light polarization. Due to the high polarization dependence in sub-micrometer SOI waveguides associated with the high index contrast, the polarization diversity approach is generally considered to be the practical solution [7], [13]. For short transmission distance as needed e.g., in data centers, the use of polarization maintaining fibers may also be a viable alternative. In both transmitter and receiver sections, it is preferable to process the signals in only one polarization, typically TE.

Despite encouraging research outcomes in monolithic light sources using Ge or doped silicon [38], [39], a commercially viable laser based on group IV materials is still a distant prospect. Current development efforts are focused on using lasers made 
of III-V semiconductors. The lasers can be coupled to the silicon PICs by flip-chip bonding techniques at the die level [16]. A wafer level approach by bonding the III-V wafer at selective areas on the SOI substrate and then proceeding with laser fabrication has also been demonstrated by a number of groups [12], [28], [29]. In this contribution we limit our discussion to the latter case which facilitates wafer level fabrication.

PICs are pursued for optical communications to overcome the disadvantages of a discrete architecture, namely size, cost, power consumption and reliability. With the compact device size and potentially high level of integration enabled by the high index contrast in silicon waveguides, the cost of the PICs is largely determined by the fabrication facilities required, the complexity of the processes and the yield of all fabrication steps [40]. Due to insufficient control in the waveguide dimensions using current fabrication technologies that result in variability in the optical phase within devices, wavelength dispersive components are generally biased to the desired operating point by thermal tuning or using a p-i-n junction [34], [41], [42]. Device power consumption is therefore not only determined by the efficiencies of signal modulation and detection, it is also strongly affected, and at times even dominated, by the power necessary for biasing. Optical losses in waveguide transmission and laserchip-fiber couplings increase the required laser output, further contributing to the overall power budget. In the following, design considerations to meet these demands and the current status for key transceiver components are reviewed. Since a large fraction of the $\mathrm{R} \& \mathrm{D}$ work in silicon photonic has been centered in the $1550 \mathrm{~nm}$ wavelength range, we limit our discussions to this case only. Recently there is an emergence of interest for the $1310 \mathrm{~nm}$ wavelength range for applications in data centers. Even though the spirit of our investigation still holds, the preferred silicon thickness will obviously differ.

\section{PASsive Components}

Various filtering and routing functions can be realized using basic building blocks such as directional couplers, multi-mode interferometers, micro-ring resonators, Mach-Zehnder interferometers, arrayed waveguide gratings (AWGs) and echelle gratings. In these devices important figures-of-merit include low insertion loss, precise channel wavelength and channel spacing, high extinction ratio, and high polarization discrimination. Tight waveguide bends with low radiation losses are needed for achieving compact device size and providing the desired free spectral range in some components such as ring resonators. All these properties are largely controlled by the waveguide cross-sectional geometry. In particular, the propagation loss is dominated by the modal overlap with interface roughness, and the accuracy of the operating wavelength is dominated by the waveguide size control.

There have been prior attempts to provide guidelines for global parameter optimization of silicon waveguide resonators based on similar design criteria: sufficiently large feature size, low sensitivity to dimensional variations, low loss and efficient thermo-optic tuning [43], [44]. The conclusion for TE polarization differs considerably from the conventional $2: 1$ as- pect ratio typically used at present, identifying waveguides of $600 \times 110 \mathrm{~nm}$ and $455 \times 265 \mathrm{~nm}$ as the optimal choice for TE and TM respectively. Evidently, that recommendation for TE has not been adopted and is not appropriate for all class of devices. For example, a silicon thickness of $110 \mathrm{~nm}$ is not suitable for devices such as modulators which use rib structures for electric contacts. It is interesting to note that the identified structure for TM polarization coincides with a number of other works on biosensing and PRs and splitters [18], [45].

In designing an integrated photonic circuit consisting of different components, there are a multitude of possibilities to handle the different trade-offs. It is not possible to single out a particular waveguide design that will optimize all components. On the other hand, there are only a few waveguide cross-sections that are widely used by the silicon photonics community. In the following, we compare the properties of these waveguides with three silicon thickness values which are the most common, i.e., 220, 250-260, and $300 \mathrm{~nm}$.

\section{A. Modal and Polarization Properties of Waveguides}

Silicon wire waveguides, where the sidewalls are etched to the buried oxide layer, have been the most commonly used for signal routing. The requirement for single mode operation limits the width of wire waveguides to below a few hundred nanometers. In this range, the effective indices are very sensitive to dimensional variations. In order to obtain acceptable control of the effective index and reduce the propagation loss while maintaining a small bend radius, rectangular wire waveguides with an aspect ratio of 2:1 are generally used for constructing the device portion of the circuits.

Electric field distributions of a wire waveguide are shown in Fig. 2(a) and (b) for the TE and TM (transverse-magnetic) polarizations, respectively. Clearly, the TE mode has a large overlap with the sidewalls, while the TM mode has a large overlap with the top and bottom interfaces. Effective indices $n_{\text {eff }}$ of the TE and TM modes as a function of waveguide width $\mathrm{W}$ are shown in Fig. 2(c) and (d) for waveguide height $\mathrm{H}$ of 220 and $260 \mathrm{~nm}$, respectively. For an aspect ratio of $\sim 2: 1$ as typically used, the fundamental TE mode is better confined than TM, having a larger effective index. For waveguides with $\mathrm{H}=220 \mathrm{~nm}$ in a symmetric environment $\left(\mathrm{SiO}_{2}\right.$ as the upper cladding), the $\mathrm{TM}$ mode is supported theoretically for a waveguide as narrow as $\mathrm{W} \sim 200 \mathrm{~nm}$. However $\mathrm{n}_{\mathrm{eff}}$ is close to the index of the buried oxide layer and the guiding is weak. When the upper cladding has a different index than that of the buried oxide layer, or if the waveguide is in a small bend, guiding can be easily lost. For example for a waveguide with air cladding, TM is cut-off for $\mathrm{W}<350 \mathrm{~nm}$ [46]. For devices with $\mathrm{SiO}_{2}$ cladding, only a radius of large than $30 \mu \mathrm{m}$ can ensure a low bend loss [64]. It was shown in early studies that TE is really the only useful polarization for this wire height, and it has been used extensively since [47]. Most foundries use this silicon thickness and supply input grating couplers for TE polarization. For larger silicon thickness, such as those shown in Fig. 2(d) with $\mathrm{H}=$ $260 \mathrm{~nm}$, both TE and TM polarizations are well guided and can support a waveguide bend radius of $5 \mu \mathrm{m}$ with low loss. A range 

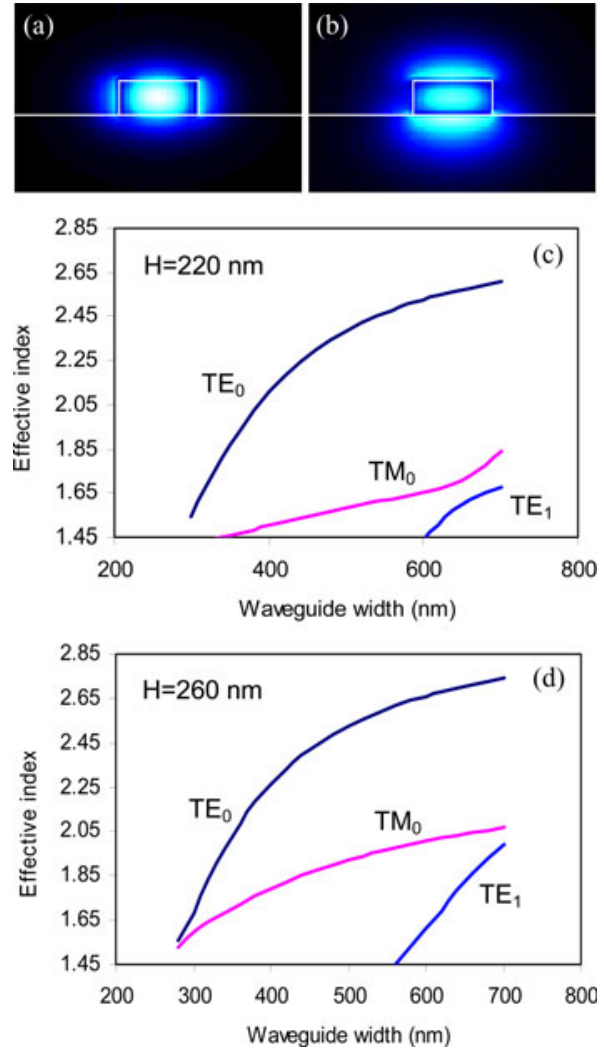

Fig. 2. Electric field distribution of silicon waveguides with a height $\mathrm{H}=$ $220 \mathrm{~nm}$ and a width $\mathrm{W}=450 \mathrm{~nm}$ : (a) TE and (b) TM. The effective indices as a function of the waveguide width for (c) $\mathrm{H}=220 \mathrm{~nm}$, and (d) $\mathrm{H}=260 \mathrm{~nm}$. The wavelength is $1550 \mathrm{~nm}$.

of components including modulators, PRs/splitters and biosensors, with high performance employing either TE or TM mode, have been demonstrated using this slightly larger silicon thickness [18], [22], [25], [48], [49]. For wire waveguides with $\mathrm{H}=$ $300 \mathrm{~nm}$, higher order modes are supported for $\mathrm{W}>320 \mathrm{~nm}$. Typically, rib waveguides with partially etched sidewalls are used.

\section{B. Phase Accuracy and Device Reproducibility}

For all interferometric optical components, accurate control of the waveguide propagation constant (i.e., phase) is the key for achieving high extinction ratio and reproducible device spectral performance. In silicon waveguides, the core material, being single crystalline silicon, has highly uniform material index. Therefore phase control in silicon devices essentially translates to controlling the waveguide cross-sectional and horizontal dimensions.

For SOI wafers commonly used in silicon photonics such as Unibond wafers by SOITEC, the silicon thickness of 150 and $200 \mathrm{~mm}$ wafers is only guaranteed to $3 \sigma= \pm 10 \mathrm{~nm}$ as stated in the product specifications [51]. Recent wafer mapping found the uniformity to be somewhat better, with a $3 \sigma$ non-uniformity of $\pm 6 \mathrm{~nm}[50]$ across the wafer. The wafer fabrication technology continues to improve. For $300 \mathrm{~mm}$ wafers which have recently been used for silicon photonics, the uniformity has reached a $3 \sigma$ of $\pm 1 \mathrm{~nm}$ [35], [54]. These data are summarized in Table I.
TABLE I

STATISTICAL VARIATIONS $(3 \sigma)$ FOR THE BEST-IN-Class SOI WAFERS AND SILICON PHOTONICS FABRICATION TECHNOLOGIES

\begin{tabular}{|c|c|c|}
\hline Wafer diameter & $200 \mathrm{~mm}$ & $300 \mathrm{~mm}$ \\
\hline Lithography & $193 \mathrm{~nm}$ dry litho & $193 \mathrm{~nm}$ immersion litho \\
\hline $\begin{array}{c}\text { Linewidth } \\
\text { uniformity }\end{array}$ & $\pm 7.8 \mathrm{~nm}[52,53]$ & $\pm 7.6 \mathrm{~nm} \mathrm{[54]}$ \\
\hline $\begin{array}{c}\text { Within-wafer } \\
\text { thickness } \\
\text { uniformity }\end{array}$ & $\pm 6 \mathrm{~nm}[52,62]$ & $\pm 1 \mathrm{~nm}[35,54]$ \\
\hline
\end{tabular}

This uniformity has come close to that for SOI wafers used for microelectronics $(3 \sigma= \pm 0.9 \mathrm{~nm})$, even though for much thicker silicon layers [51].

The in-plane dimensions of waveguides are determined by the mask technology, the lithography tool and etching processes. In current foundry services, DUV dry lithography using 248 or $193 \mathrm{~nm}$ light sources are available for $200 \mathrm{~mm}$ wafers. The size of CD (critical dimension) features is typically controlled to a $3 \sigma$ accuracy of $\pm 8 \mathrm{~nm}$ in $193 \mathrm{~nm}$ lithography [52], [53]. Linewidth uniformity for $248 \mathrm{~nm}$ lithography is considerably worse [52]. For other features of different widths and gaps, especially between areas with different pattern densities, dimensional controls are more challenging due to proximity effects. For either e-beam or DUV lithography, size deviations of up to $\pm 10 \mathrm{~nm}$ from design are not uncommon. A few research and pre-commercial facilities have started processing silicon photonics circuits using $300 \mathrm{~mm}$ wafer fabs where $193 \mathrm{~nm}$ immersion lithography becomes available. Although the minimum CD is reduced $80 \mathrm{~nm}$ [35], the results reported so far only showed marginal improvement in absolute linewidth uniformity as summarized in Table I [53], [62]. This comes far short of the more than $\times 5$ times improvement seen in the silicon thickness uniformity. Furthermore, these facilities are not yet publicly available. Coming hand-in-hand with higher precisions, such infrastructures are also much more costly. Wide spread use of such facilities requires even higher product volume to justify.

The resonance wavelength of a ring resonator can be used to characterize the phase sensitivity of the constituent cavity waveguide. Fig. 3 shows the spectral wavelength shift with respect to dimensional changes, defined as $\delta \lambda / \delta \mathrm{W}=\left(\lambda / \mathrm{n}_{g}\right) \delta \mathrm{n}_{\text {eff }} / \delta \mathrm{W}$ and $\delta \lambda / \delta \mathrm{H}=\left(\lambda / \mathrm{n}_{g}\right) \delta \mathrm{n}_{\text {eff }} / \delta \mathrm{H}$, where $\lambda$ is the wavelength and $\mathrm{n}_{g}$ is the waveguide group index. For waveguides with typically used aspect ratio of $2: 1$, the TE mode is more sensitive to width variations, while the TM mode is more sensitive to height variations as one naturally expects. The expected $3 \sigma$ variability in the resonance wavelength for resonators made by state-ofthe-art $193 \mathrm{~nm}$ immersion lithography on $300 \mathrm{~mm}$ wafers is summarized in Table II for waveguide structures typically used. The linewidth and thickness uniformity data in Table I are used for these estimations. Clearly, such variations are still unsatisfactory for meeting the spectral requirements of most dispersive devices, not to mention integrating a large number of components. Currently, spectral tuning using thermo-optic effect or current injection in p-n junctions is used to mitigate the problem. The associated power consumption and design complexity, 
TABLE II

EXPECTED WAVELENGTH $3 \sigma$ SCATter $\Delta \lambda_{3} \sigma$ (IN NANOMETERS) DUE TO DiMENSIONAL VARIATIONS FOR DifFERENT WAVEGUide StRUCTURES MADE USING STATE-OF-THE-ART 193 nm IMMERSION LitHOGRAPHY

\begin{tabular}{|c|c|c|c|c|}
\hline Wire structure & \multicolumn{2}{|c|}{$\Delta \lambda_{3 \sigma}$ (Wire-TE) } & \multicolumn{2}{c|}{$\Delta \lambda_{3 \sigma}$ (Wire-TM) } \\
\hline $\mathrm{H} / \mathrm{W}(\mathrm{nm})$ & $\Delta \lambda_{3 \sigma}(\mathrm{W})=7.6 \times \Delta \lambda / \Delta \mathrm{W}$ & $\Delta \lambda_{3 \sigma}(\mathrm{H})=1.0 \times \Delta \lambda / \Delta \mathrm{H}$ & $\Delta \lambda_{3 \sigma}(\mathrm{W})=7.6 \times \Delta \lambda / \Delta \mathrm{W}$ & $\Delta \lambda_{3 \sigma}(\mathrm{W})=1.0 \times \Delta \lambda / \Delta \mathrm{H}$ \\
\hline $220 / 450$ & \pm 6.6 & \pm 1.4 & \pm 2.8 & \pm 2.7 \\
\hline $260 / 450$ & \pm 6.4 & \pm 1.1 & \pm 3.0 & \pm 2.7 \\
\hline
\end{tabular}

\begin{tabular}{|l|c|c|c|c|c|}
\hline \multicolumn{6}{|l|}{ Rib structure - TE } \\
\hline $\mathrm{H} / \mathrm{W} / \mathrm{D}$ & $\begin{array}{c}\text { Minimum } \\
\text { radius }(\mu \mathrm{m})\end{array}$ & $\begin{array}{c}\text { Min. loss } \\
(\mathrm{dB} / \mathrm{cm})\end{array}$ & $\Delta \lambda_{3 \sigma}(\mathrm{W})=7.6 \times \Delta \lambda / \Delta \mathrm{W}$ & $\Delta \lambda_{3 \sigma}(\mathrm{H})=1.0 \times \Delta \lambda / \Delta \mathrm{H}$ & $\Delta \lambda_{3 \sigma}(\mathrm{D})=5 \times \Delta \lambda / \Delta \mathrm{D}$ \\
\hline $220 / 700 / 70$ & 25 & $0.12[61]$ & \pm 0.8 & \pm 1.9 & $-/+1.8$ \\
\hline $300 / 360 / 150$ & $10[55]$ & $2[55]$ & \pm 3.0 & \pm 1.5 & $-/+6.2$ \\
\hline
\end{tabular}

These values are obtained using simulated $\Delta \lambda / \Delta \mathrm{H}, \Delta \lambda / \Delta \mathrm{W}$ and $\Delta \lambda / \Delta \mathrm{D}$, together with the dimensional uniformity data from Table I (i.e., $\Delta \mathrm{W} 3 \sigma= \pm 7.6 \mathrm{~nm}$ and $\Delta \mathrm{H}_{3 \sigma}= \pm 1 \mathrm{~nm}$ ). An etch depth control of $\Delta \mathrm{D}_{3 \sigma}= \pm 5 \mathrm{~nm}$ is assumed. Note that TM is only weakly guided for waveguides with a height of $220 \mathrm{~nm}$.
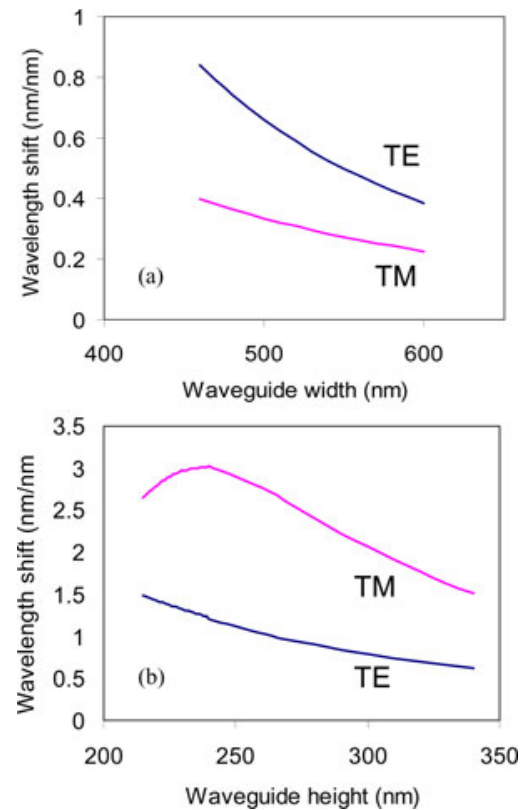

Fig. 3. (a) Wavelength shift $\delta \lambda / \delta \mathrm{W}$ as a function of waveguide width for $\mathrm{H}$ $=260 \mathrm{~nm}$; (b) Wavelength shift $\delta \lambda / \delta \mathrm{H}$ as a function of waveguide height for $\mathrm{W}=450 \mathrm{~nm}$. Here, the upper cladding is air and the wavelength is $1550 \mathrm{~nm}$.

however, pose severe limits on the transceiver performance and cost.

Different strategies can be pursued to reduce the tuning power. Comparing between the TE and TM polarizations with their respective limitations as shown in Fig. 3, we observe that $(\delta \lambda / \delta \mathrm{H})_{\mathrm{TM}} \sim 3(\delta \lambda / \delta \mathrm{W})_{\mathrm{TE}}$. This relation suggests that when the achievable dimension control is $\delta \mathrm{H}<3 \delta \mathrm{W}$, the use of TM polarization gives a higher degree of phase control. This requirement is already achieved for uniformity in $300 \mathrm{~mm}$ wafer technology. Furthermore, the thickness of the wafers can be mapped and corrected over the wafer by using location specific processing [57]. Although such thickness correction of SOI wafers requires additional processing, it is still beneficial for achieving systems with low thermal tuning power. To improve the phase precision of the TE polarization, however, requires even more stringent dimensional control than currently achieved in $193 \mathrm{~nm}$ immersion lithography, which appears to be unrealistic within the medium term. The control of in-plane dimensions is ultimately limited by the accuracy of the processing and metrology tools, which is presently at $2 \mathrm{~nm}$. Given the recent development, we believe an even higher precision of silicon thickness control at the wafer manufacture source is at least a plausible improvement that may be achieved in the near or medium term.

Another strategy of improving phase accuracy is by introducing rib waveguides in the photonic circuits. Due to coupling to the slab TE mode, the TM polarization is not well supported in such geometries unless the etch is deep [58]. Both the rib width and the etch depth can be adjusted to alter the degree of modal confinement of the TE mode and the corresponding phase sensitivity. For signal transport, multi-mode straight waveguides can be adopted, but only single-mode waveguides are used in phase sensitive sections [56]. This imposes a tradeoff between bend radius and phase sensitivity. Detailed characterization of two particular single-mode rib geometries has been reported, namely for waveguides with a height/width/etch depth of 220/450/70 nm [56] and 300/360/150 nm [36]. The corresponding calculated phase sensitivities are indicated in Table II. An etch depth precision of $\pm 5 \mathrm{~nm}$, now routinely obtained for $300 \mathrm{~mm}$ technology, is used. This represents a higher level of control compared to the $\pm 10 \mathrm{~nm}$ variations observed in an $200 \mathrm{~mm}$ technology [8]. One can observe that the tolerance to width variations is improved in both structures compared to that for wires, but the calculated tolerance to the $\pm 5 \mathrm{~nm}$ etch depth variation demonstrates that etch depth uniformity is an important issue for rib waveguides. In particular, for the case of $300 / 360 / 150$ waveguides, the etch depth sensitivity is comparable to the width sensitivity of wires. It is the aspect ratio, not merely the rib height that determines the phase sensitivity [44].

\section{Waveguide Propagation Loss, Back Reflection and Bend Loss}

In silicon waveguides, propagation loss is mainly due to scattering from the roughness at the core-cladding interfaces. In commonly used SOI wafers, the silicon surface is atomically smooth with an RMS of less than $0.1 \mathrm{~nm}$. Waveguide sidewall roughness is determined by the lithography and etching processes and is typically on the order of a few nanometers, much larger than the surface roughness [59]. Even though postprocessing steps such as re-oxidation of the waveguides have been used to reduce sidewall roughness, and fabrication technologies continue to improve (e.g., the use of immersion lithography), scattering remains the dominant cause of loss in silicon 
waveguides. For fully etched wire waveguides, the TE mode suffers a higher loss than TM because of its larger overlap with sidewall roughness [60]. For example, propagation losses of 2.4 and $0.59 \mathrm{~dB} / \mathrm{cm}$ are obtained for TE and TM mode, respectively, for waveguides made using $0.13 \mu \mathrm{m}$ CMOS technology $(\mathrm{H}=$ $220 \mathrm{~nm}$ ) [61]. This trend has been observed experimentally for $\mathrm{H}$ of $200 \mathrm{~nm}$ to $260 \mathrm{~nm}$, showing the loss for TE is approximately 2 to 4 times of that for TM. Using $300 \mathrm{~mm}$ wafer technology (see Table I), the loss for TE has recently been reduced to a record low value of $0.7 \mathrm{~dB} / \mathrm{cm}$ [62]. It should be expected that this improvement in sidewall roughness would also lower the loss of the TM mode proportionally.

Based on the argument of modal overlap with sidewall roughness, the loss in single mode wire waveguides with larger $\mathrm{H}$ is expected to be larger, but there has not been a direct experimental comparison reported. A number of studies have used rib waveguides with shallow etch to reduce the loss significantly. The modal confinement, controlled by both the etch depth and rib width, determines the overall loss. For single mode waveguides with $\mathrm{H} / \mathrm{W} / \mathrm{D}$ of $220 / 700 / 70 \mathrm{~nm}$, a low loss of $0.12 \mathrm{~dB} / \mathrm{cm}$ has been achieved using the $300 \mathrm{~mm}$ technology [62]. In waveguides with $\mathrm{H}=300 \mathrm{~nm}$ made using the $200 \mathrm{~mm}$ technology, single mode waveguides of $360 \mathrm{~nm}$ width exhibited a loss of $2 \mathrm{~dB} / \mathrm{cm}$ (after full CMOS processing), while $3 \mu \mathrm{m}$ wide multimode waveguides showed a loss as low as $0.026 \mathrm{~dB} / \mathrm{cm}$ (both with an etch depth of $150 \mathrm{~nm}$ ) [36]. The price one has to pay for achieving these low losses is a much larger bend radius for using this type of geometry, or more complex layouts with rib-to-wire transitions, giving these structures each its own appropriate utility [56].

Back reflection can generate a variety of impairments in optical systems such as crosstalk and return loss degradation. This can severely hinder the application of silicon photonic components in many practical applications, although the reflection is only a small contribution to the forward transmission loss. Similar to forward propagation loss in waveguides, back reflection originates from sidewall roughness. Therefore they share similar characteristics and can be mitigated using similar strategies. For waveguides with a typical aspect ratio of 2:1, TE polarization experiences higher backscattering than TM by as much as $20 \mathrm{~dB}$ [60]. Backscattering can be resonantly enhanced in devices such as ring resonators and becomes the limiting factor for device performance [63]. This limits the quality factor to below $10^{4}$ and resonance splitting is common. When the TE polarization is used in the circuit, one mitigation strategy is to locally use a resonant cavity in TM mode as demonstrated in [64]. However the design is complex and the waveguide dimension control is stringent. Alternatively, rib waveguides can be used with a larger bend radius. A simpler option is to adopt the TM mode as the general polarization in wire waveguides using a silicon thickness where the mode is well supported (e.g., $\mathrm{H} \sim 250 \mathrm{~nm})$.

There has been extensive simulation work which shows that the bend loss for the TE mode is lower than that for TM polarization in wire waveguides [65]-[67]. Nonetheless for radii of larger than $5 \mu \mathrm{m}$, both polarizations give minimum bending loss [68]-[71], and high performance resonators have been

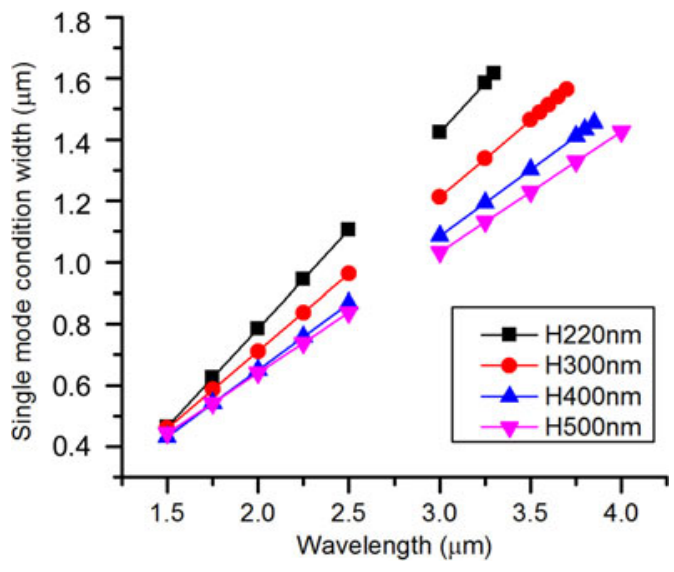

Fig. 4. Single mode width versus wavelength for SOI strip waveguides with different heights.

demonstrated in TM polarization with a radius as small as $2 \mu \mathrm{m}$ [71]. This range of radii can satisfy most circuit layout requirements.

Silicon is transparent not only in the near-infrared (NIR) but also up to a wavelength of $\lambda \sim 8 \mu \mathrm{m}$ and is potentially a viable material for mid-infrared (MIR) applications. Therefore, in addition to the intensive NIR research activity, MIR silicon photonics has become an increasingly interesting research area [72]. The MIR is a spectral region of tremendous scientific and technological interest. Spanning a large wavelength range (2-20 $\mu \mathrm{m})$, it contains strong vibrational signatures for a number of gases and molecules, and hence photonic devices that operate in this region can be potentially applied for a host of applications in gas, environmental, and bio-chemical sensing, industrial process control, defence and security sectors, or point of care diagnostics.

Silicon dioxide, which usually serves as a bottom cladding for NIR silicon photonic devices, has a high material loss for $\lambda$ between 2.6 and $2.9 \mu \mathrm{m}$, and beyond $\lambda \sim 4 \mu \mathrm{m} \mathrm{[73].} \mathrm{SOI} \mathrm{is}$ a desirable photonic material platform in the MIR regions with low $\mathrm{SiO}_{2}$ absorption due to mature fabrication processes and the high material quality achieved over the last 20 years. To date, for the 2-4 $\mu \mathrm{m}$ wavelength range, different SOI thicknesses have been used: from $220 \mathrm{~nm}$ in [74] for wavelengths around 2.3 to $340 \mathrm{~nm}$ in suspended structures [75], to 400 and $500 \mathrm{~nm}$ in [76], [77]. To identify how waveguides of different thicknesses perform, we have simulated SOI wire waveguides across the 1.5-4 $\mu \mathrm{m}$ wavelength range

Fig. 4 shows the maximum width for single mode propagation for wire SOI waveguides with different silicon thicknesses for TE polarization. Points are plotted only where there is single mode propagation and propagation loss is below $2 \mathrm{~dB} / \mathrm{cm}$, thus representing the transparency ranges of the platform. In the simulations, which do not take into account scattering losses, the cut-offs at longer wavelengths are mostly due to radiative losses stemming from waveguide geometry. For TM polarization, the dependence on silicon thickness is more severe, resulting in lower transparency ranges than for TE polarization. It can be seen from Fig. 4 that thicker Si layers can exploit the full $\mathrm{SiO}_{2}$ 

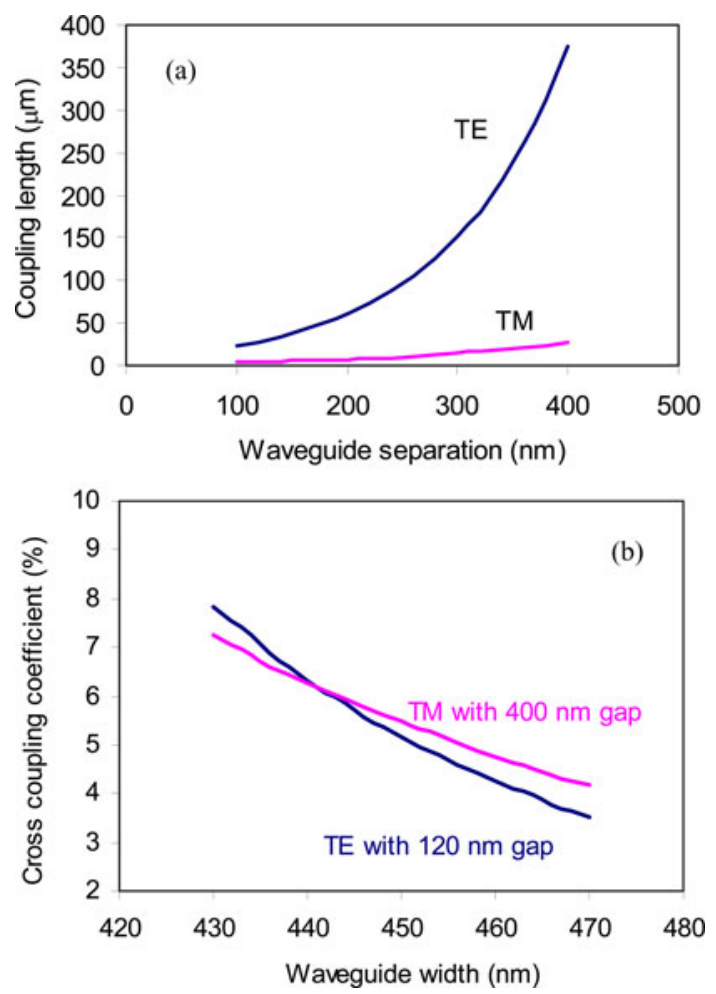

Fig. 5. (a) Coupling length $L_{\pi}$ for full power transfer as a function of waveguide separation. Here, $\mathrm{H}=260 \mathrm{~nm}, \mathrm{~W}=450 \mathrm{~nm}$, the wavelength is $1550 \mathrm{~nm}$ and air is the upper cladding. (b) Cross-coupling coefficient as a function of the waveguide width, with the center-center separation kept at a constant.

transparency range up to $4 \mu \mathrm{m}$, and therefore they would be preferred platforms for the MIR. If foundry fabrication for such SOI wafers becomes more readily available, it can accelerate the penetration of silicon photonics into other product areas beyond optical communications.

\section{Evanescent Coupling Strength and Losses}

Directional couplers are commonly used as beam splitters in a variety of devices such as Mach-Zehnder interferometers and ring resonators. This device relies on the evanescent coupling of optical modes between two closely spaced waveguides. The coupling strength can be characterized by a coupling length $L_{\pi}$ which is the length for full power transfer from one waveguide to the other [78]. For a coupler of physical length $L_{C}$, the power cross-coupling coefficient is $\kappa^{2}=\sin \left(\pi L_{C} / 2 L_{\pi}\right)$. An example of the coupling length as a function of the waveguide center-center separation is shown in Fig. 5(a). The coupling between TM modes is much stronger than that for TE at the same waveguide separation, with $L_{\pi}^{\mathrm{TE}} \sim 10 L_{\pi}^{\mathrm{TM}}$.

To achieve a power cross-coupling coefficient of $\kappa^{2} \sim 5 \%$ using a coupler of $4 \mu \mathrm{m}$ length with $\mathrm{H}=260 \mathrm{~nm}$, the gap size required is $110 \mathrm{~nm}$ and $400 \mathrm{~nm}$ for TE and TM respectively. To obtain a similar coupling in $\mathrm{TE}$ for $\mathrm{H}=220 \mathrm{~nm}$, the gap size is $140 \mathrm{~nm}$. Obviously, a much narrower gap is required for TE polarization than for TM to achieve a given coupling strength. The fabrication of a TE directional coupler is therefore more challenging, suffering from proximity effects in the lithography process. Additionally, a significant mode-conversion loss occurs in resonators with small bend radius. A gap size of below $120 \mathrm{~nm}$ [79] or $200 \mathrm{~nm}$ [80] has been observed to cause significant coupling losses, depending on the bend radius used. By using the TM mode, this problem can be alleviated since a much larger gap can be used.

The coupling coefficient is also strongly affected by small dimensional variations or incomplete etching in the gap. If the etching is complete, the coupling variation generally comes from the waveguide width fluctuations. The center-center separation between the waveguides can be considered accurate. In this case, the coupling coefficient as a function of the waveguide width is shown in Fig. 5(b). The TM polarization is also observed to be slightly more tolerant.

\section{E. Grating Couplers}

Grating coupling has been developed rapidly in the last few years for coupling light between optical fibers and silicon submicron-sized waveguides. Compared to the butt-coupling approach, grating couplers can be located anywhere on the chip with an out-of-plane coupling scheme. This increases significantly the flexibility in the circuit design, and also enables wafer-scale automated testing. Grating couplers also generally have larger tolerances in fabrication and alignment process than butt-coupling approaches. Here we focus on an analysis of the coupling efficiency, which is the key criterion for most applications. We found that the best coupling efficiency achievable for grating couplers is largely affected by the waveguide thickness.

For ease of analysis, we consider only light traveling from grating to fiber. The efficiency of light that is coupled in the opposite direction should be the same due to reciprocity. In order to maximize the coupling efficiency of a grating coupler, three issues have to be addressed. The first is eliminating the back reflection from the grating. The second is increasing the mode matching efficiency between the grating mode and the mode in the optical fiber. These two can be addressed by proper design of the grating structures, such as apodization [81]-[83], without complicating the fabrication process. The last one is enhancing the directionality, which is defined as the optical power coupling upwards towards the fibre, as a fraction of the total coupled power. Typically, a significant portion of the light would be coupled into the substrate and lost. The back reflection and mode matching efficiency can generally be optimized to values approaching zero and unity, respectively, for most common SOI waveguide geometries by careful grating design. The overall coupling efficiency would then be limited only by the directionality. Theoretically, one way to improve directionality is by adding a metal or dielectric mirror underneath the grating coupler [84]. However, this is difficult to realize in practice. The optical resonance between the mirror and grating can also compromise the achievable optimum coupling efficiency.

A simple and effective way to enhance the directionality of the grating coupler is to optimize the etch depth of the grating, as has been proposed and demonstrated for micrometric SOI rib waveguides [85]. The directionality would be improved when there is constructive interference for coupling upwards and 


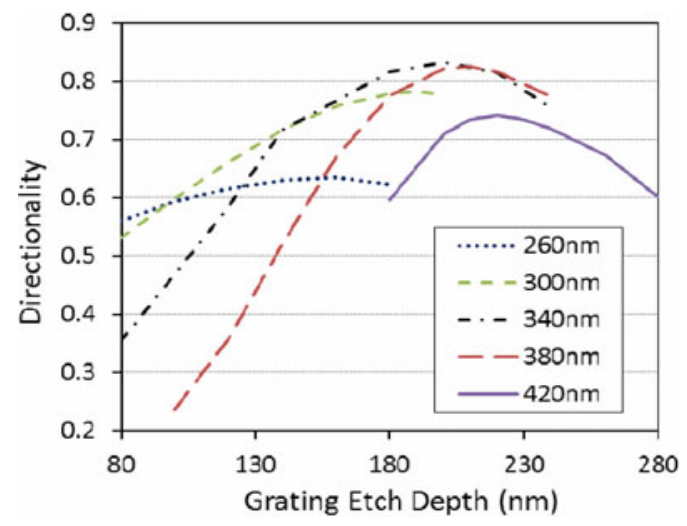

Fig. 6. Calculated directionality of gratings against grating etch depths for SOI platforms with various waveguide thicknesses.

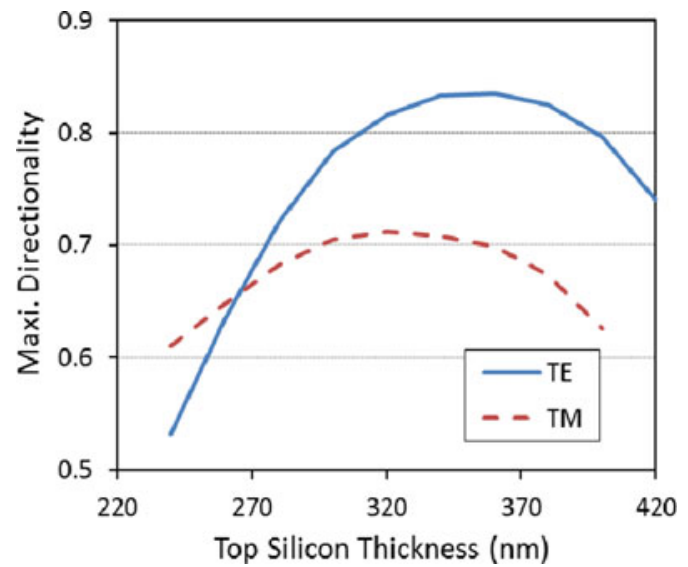

Fig. 7. The best achievable directionality for SOI platforms with various waveguide thicknesses for both TE and TM polarizations.

destructive interference for coupling downwards to the substrate. Here we perform two-dimensional FDTD simulations to calculate the directionality of gratings for SOI platforms with various waveguide thicknesses in the submicron range, and the results are plotted in Fig. 6. The directionality is found to change approximately sinusoidally with respect to the etch depth. More importantly, the best directionality achievable varies significantly for different waveguide thicknesses. We assume coupling to a 10 degree titled single-mode fiber (with $10.4 \mu$ m mode field diameter). The waveguide grating is embedded in a top oxide cladding, and the silicon substrate is not considered in the simulations. Therefore, no light interference from the buried oxide (BOX) layer is included. The fill factor of the grating is about 0.4 , which is defined as the ratio of the shallow-etched groove width to the grating period. We find that the fill factor has negligible effect on the directionality. The grating periods are calculated for every combination of the top silicon thickness and grating etching depth according to the phase matching equation, to ensure a peak coupling wavelength of $1550 \mathrm{~nm}$. Only the TE mode is considered in Fig. 6.

More simulations are carried out to search for the best achievable directionality for SOI platform with various waveguide thicknesses and both TE and TM polarizations. The results are plotted in Fig. 7. We can see that for TE polarization grat-

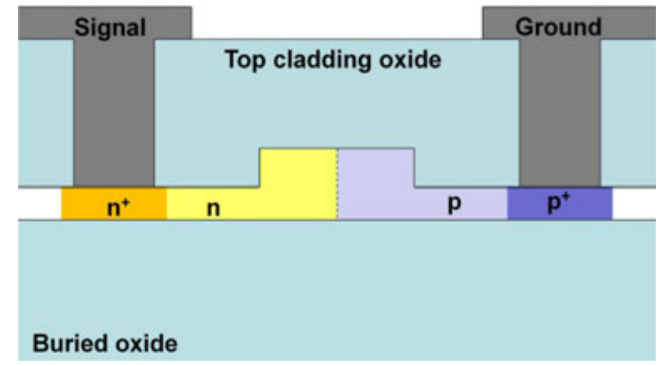

Fig. 8. Cross-sectional diagram of a typical carrier depletion modulator.

ings would have a peak directionality of 0.84 for $\sim 350 \mathrm{~nm}$ waveguide thickness. TM polarization gratings would have a peak directionality of 0.71 for $\sim 330 \mathrm{~nm}$ waveguide thickness. Although the directionality can also be improved by adopting a BOX layer with a proper thickness, or by adding dielectric layers on top, the optimization of waveguide thickness and grating etch depth gives a more significant improvement in directionality. Besides, all those parameters and techniques can be utilized at the same time to maximize performance improvement. Therefore, it should be recommended to choose a SOI platform with waveguide thickness around the peak value of the directionality as shown in Fig. 7 to optimize the coupling efficiency of grating couplers. One good example was presented in [86] where the coupling efficiency of a uniform grating was improved from $37 \%$ to $78 \%$ (or $1.5 \mathrm{~dB}$ ), by adding a $150 \mathrm{~nm}$ poly-silicon overlay to the silicon waveguide with $220 \mathrm{~nm}$ thickness. This enhancement is due to the directionality change as shown in Fig. 7. Grating coupling efficiencies of $1.5 \mathrm{~dB}$ (at $1490 \mathrm{~nm}$ wavelength) and $1.9 \mathrm{~dB}$ (at $1550 \mathrm{~nm}$ ) reported for SOI platform with $310 \mathrm{~nm}$ silicon thickness and optimized BOX thickness are supporting examples of this finding [35].

\section{CARrier Depletion Silicon Modulators}

Silicon optical modulators based upon carrier depletion have received significant research interest due to the potential for high speed operation, fabrication simplicity, and CMOS compatibility [22], [24], [87], [88]. The geometry of the waveguide in which the modulator is based has a significant impact on the device performance [89]-[91]. In this section we theoretically analyze with varying geometries the performance of the modulator in terms of its speed, loss and power consumption and polarization dependence.

The most popular configuration of carrier depletion modulator is shown in Fig. 8. It is based within a rib waveguide with the p-n junction positioned in the centre of the waveguide. Highly doped $\mathrm{p}$ and $\mathrm{n}$ type regions which are used to form ohmic contacts to the device electrodes are positioned in the slab regions to either side of the waveguide.

Even within this relatively simple design there are several variants aside from the waveguide geometry which affect the performance of the device. In this analysis we fix these variants using typical parameters as demonstrated in the literature in order to perform a more general analysis of the device performance with different waveguide geometries. The slab 
height is fixed at $100 \mathrm{~nm}$, the $\mathrm{p}$ and $\mathrm{n}$ type doping densities at $5 \times 10^{17}$ ions.cm ${ }^{-3}$, the $\mathrm{p}+$ and $\mathrm{n}+$ doping densities at $1 \times$ $10^{20}$ ions.cm ${ }^{-3}$ and rib edge to highly doped region separation at $1000 \mathrm{~nm}$.

\section{A. Power Consumption}

The parameters which dictate the device power consumption depend on how the phase modulator element is driven. If traveling wave electrodes are used as typically is the case in Mach-Zehnder devices, a termination is required after the modulator which will dissipate all of the power not consumed by the modulator. Therefore the power consumption will depend only on the drive voltage. For devices driven as a lumped element which do not use a termination resistor, both the capacitance of the device and the drive voltage are important.

In both cases therefore, a low power consumption results when a low drive voltage is used. In order to minimize the drive voltage required the modulation efficiency of the device should be optimized. A large modulation efficiency results when a large overlap between the optical mode and the region of depleting free carrier occurs. The depletion width for a device with $p$ and $\mathrm{n}$ type densities of $5 \times 10^{17}$ ions.cm ${ }^{-3}$ and a reverse bias of $2 \mathrm{~V}$ (CMOS compatible) is approximately $125 \mathrm{~nm}$. Optical modeling has been used to calculate the fraction of the total optical power propagating in waveguides which overlaps with this $125 \mathrm{~nm}$ region in the centre of the waveguide for different waveguide geometries. The results are shown in Fig. 9 for the TE and TM polarizations. For the TE polarization the modal overlap with the varying depletion tends to increase with waveguide height in the range considered with an optimal waveguide width of approximately $350 \mathrm{~nm}$. For the TM polarization the same trend in waveguide height is observed, however the optimal width is smaller $(\sim 300 \mathrm{~nm})$.

The capacitance of the device is dependent on the doping density of the $\mathrm{p}$ and $\mathrm{n}$ type regions and the height of the waveguide since the area of the capacitor is effectively increased. The capacitance of the device for different waveguide heights can be theoretically calculated for the doping densities stated above. Fig. 10 shows the theoretical junction capacitance per $\mathrm{mm}$ length at $0 \mathrm{~V}$ for different waveguide heights (and widths). Although the capacitance is not dependent on the waveguide width a 2-D plot is used to retain consistency with the other figures.

In both traveling wave and lumped element devices the power consumption has a second order dependence on the drive voltage but a linear dependence on the capacitance. The modulation efficiency is therefore the dominant factor in achieving low power consumption and therefore our results suggest that the best results occur for thicker silicon device layers as shown in Fig. 9.

\section{B. Optical Loss}

The optical loss of the device due to the doped regions of the waveguide has been calculated by running optical simulations on the structure at $0 \mathrm{~V}$ (with depletion width of approximately $70 \mathrm{~nm}$ ). The results of this analysis in $\mathrm{dB} / \mathrm{mm}$ can be seen in
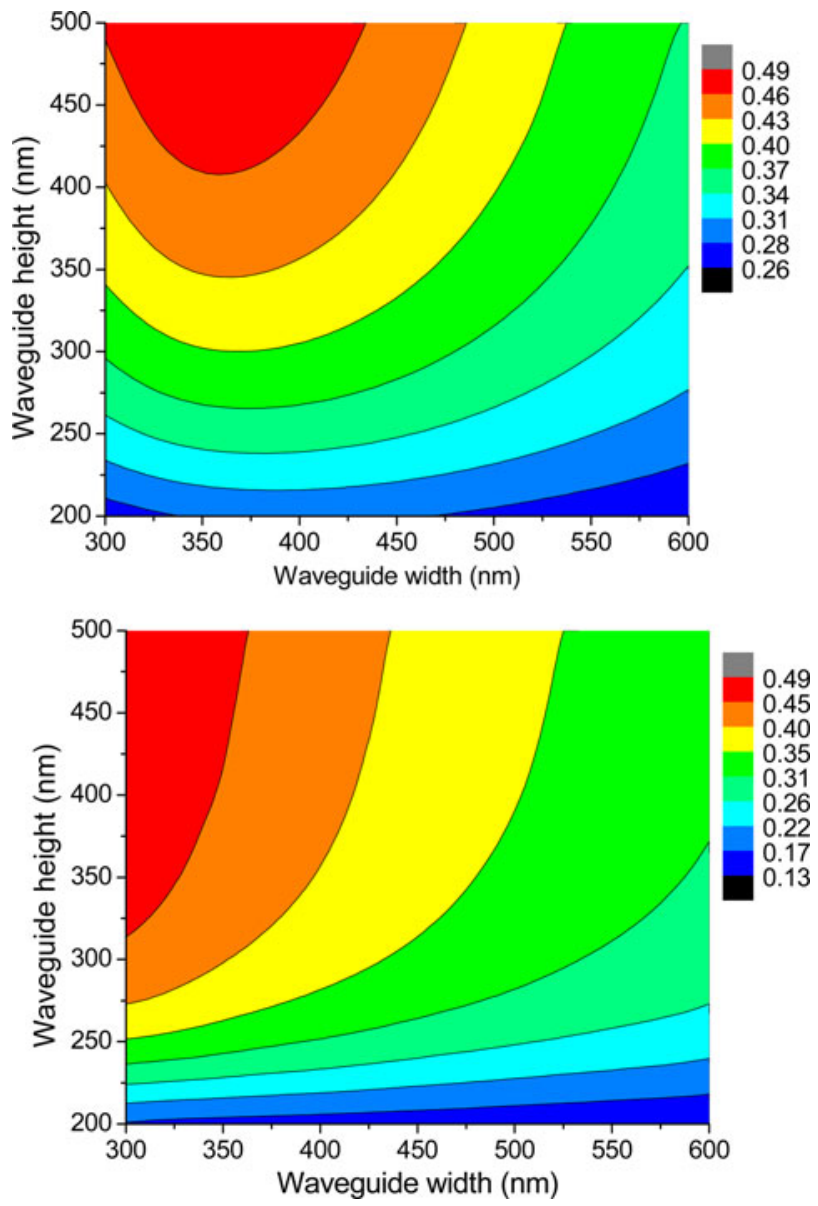

Fig. 9. Fraction of optical power propagating within a $125 \mathrm{~nm}$ slit in the middle of the waveguide for TE polarization (top) and TM polarization (bottom).

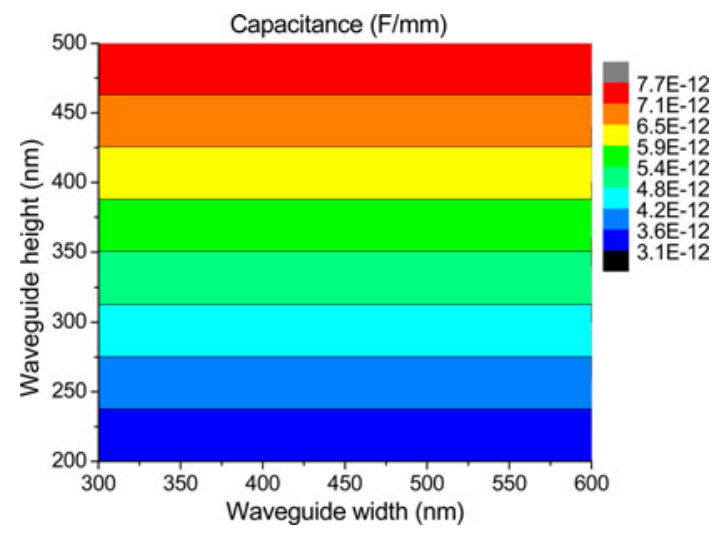

Fig. 10. Device capacitance per mm length against different waveguide geometries.

Fig. 11 for both TE and TM polarizations. In both cases the optical loss tends to increase with increasing waveguide width. For TM polarized light there is also large waveguide height dependence for values below $300 \mathrm{~nm}$.

\section{Device Speed}

The speed of the phase modulator element is usually dictated by its RC time constant. In devices driven in traveling wave 

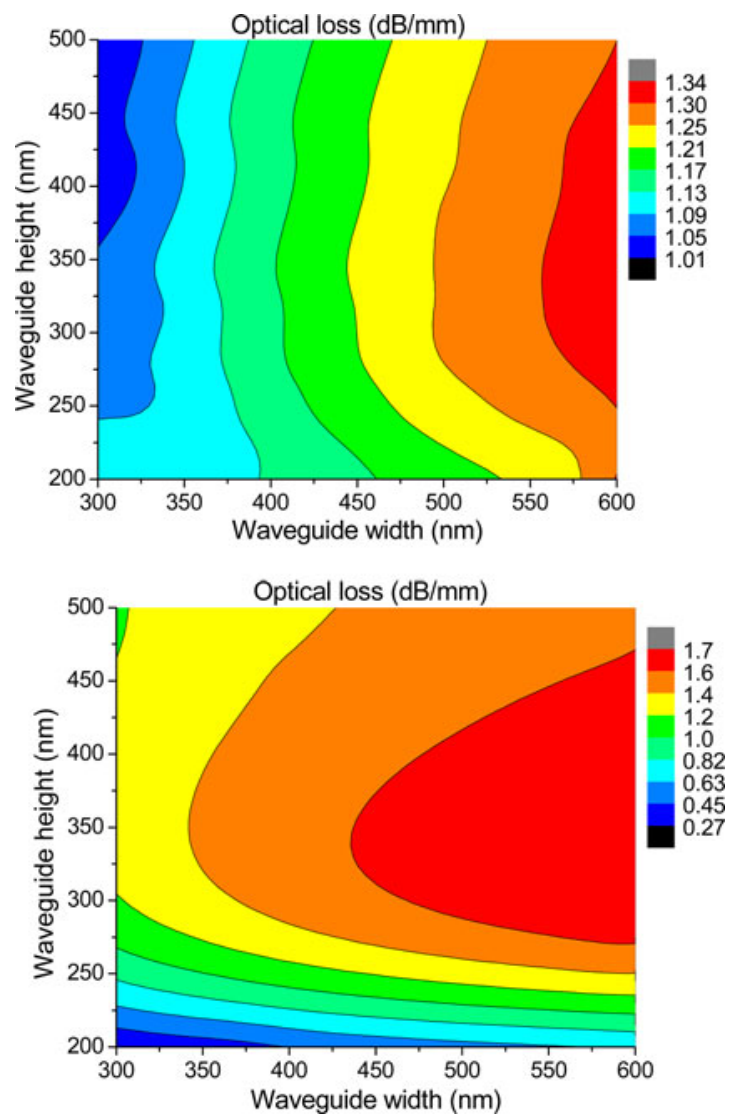

Fig. 11. Optical doping loss $(\mathrm{dB} / \mathrm{mm})$ against different waveguide dimensions for TE polarization (top) and TM polarization (bottom).

configuration a large capacitance will also restrict the length over which high speed electrical signals can be propagated along the electrodes.

The resistance element comprises contact resistance between the highly doped regions and the electrodes, and resistance through the slab and rib regions which will be dependent on the respective thicknesses and the doping configurations. The highest resistance by about an order or magnitude is usually the resistance of the $\mathrm{p}$ and the $\mathrm{n}$ type slab regions since they are both thin, having high resistivity and a relatively long electrical length. With the slab height and doping densities fixed in this analysis the total access resistance will not vary significantly with waveguide geometry. The dependence of the device capacitance on the waveguide geometry, shown in Fig. 10, suggests that the fastest devices should be based on thinner SOI.

\section{Overall Figure of Merit}

If we consider an overall figure of merit (FOM) which takes into account the speed, optical loss and power consumption we can estimate the optimal waveguide dimensions on which a carrier depletion modulator should be based. Ideally, we want high speed, low optical loss and low power consumption for a modulator. As previously discussed the FOM should have a second order dependence on the modulation efficiency since the power consumption is proportional to the square of the voltage which itself is inversely proportional to the modulation efficiency. The
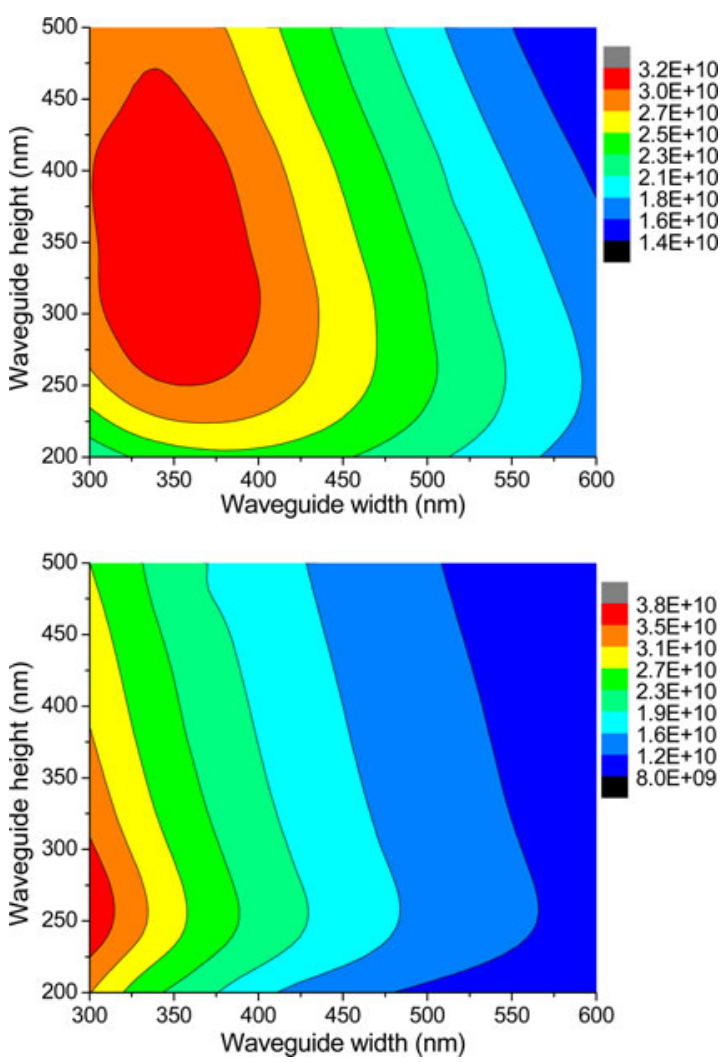

Fig. 12. FOM against different waveguide dimensions for TE polarization (top) and TM polarization (bottom).

FOM should then have a linear dependence on the optical loss and capacitance (speed). An overall FOM can therefore be calculated using the expression below.

$$
\mathrm{FOM}=\frac{(\text { Fraction of modal overlap with depleted region })^{2}}{\text { Capacitance } \times \text { Optical loss }} .
$$

Fig. 12 shows the FOM calculated for different dimensions for both TE and TM polarizations. For TE polarization the optical waveguide width and height are approximately 350 and $360 \mathrm{~nm}$, respectively. For TM polarization the optimal dimensions for low loss needs to be out of the range considered with a required width less than $300 \mathrm{~nm}$.

\section{E. Further Considerations}

1) Passive Waveguide Loss: As discussed in Section III-C the optical loss of the passive waveguides (in which the phase modulators are also fabricated) is dominated by scattering loss due to sidewall roughness. If the width of the waveguide is reduced to achieve increased modulation efficiency, the scattering loss will dramatically rise. This loss will place a minimum on the waveguide width which should be used for the modulator.

2) Polarization Independence: An optical circuit that has a performance which is not sensitive to the input polarization is advantageous in terms of ease of packaging, ultimately meaning that the cost of assembly can be reduced. For the modulator, the modulation efficiency and the optical loss should ideally be the same for both polarizations. Fig. 13 is a plot of the difference 

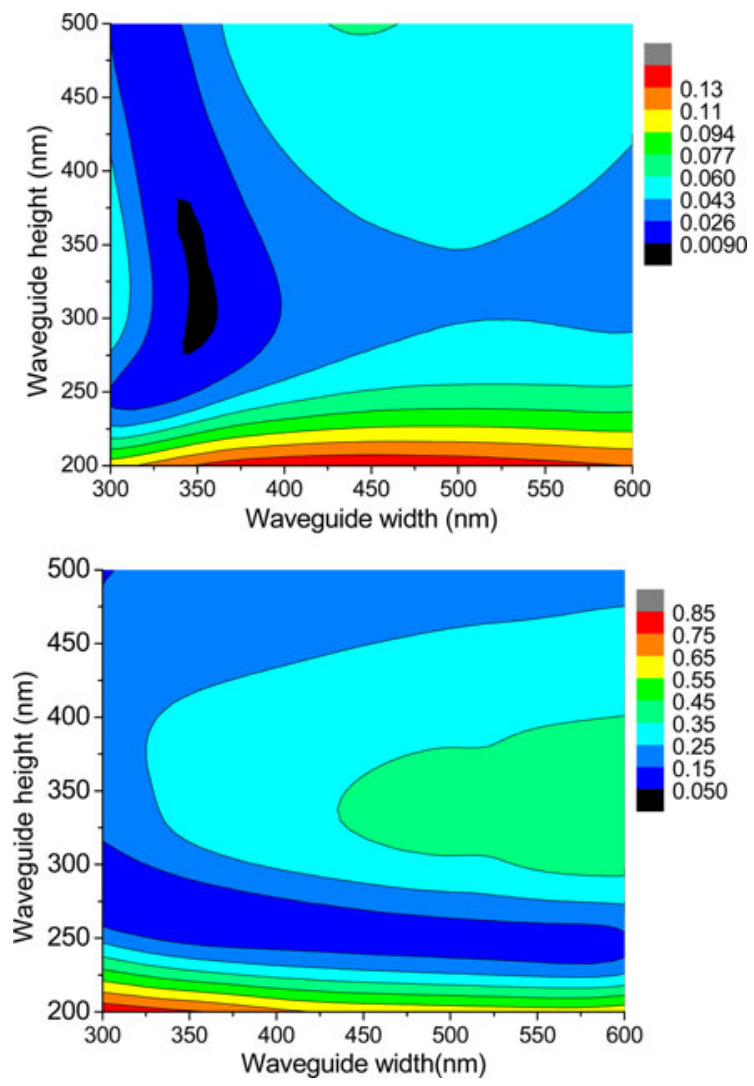

Fig. 13. Difference between the fraction of modal overlap with the varying depletion region for TE and TM polarizations (top) and difference in the optical loss in $\mathrm{dB} / \mathrm{mm}$ for TE and TM polarizations (bottom).

between the fraction of modal overlap and the difference in the loss for both polarizations. If a waveguide width of $350 \mathrm{~nm}$ and height of $300 \mathrm{~nm}$ are considered both the difference in optical loss and difference in the fraction of modal overlap are very low.

\section{WAFER SCALE INTEGRATION OF LASERS}

Although direct monolithic growth on silicon of III-V direct bandgap semiconductors [92] or strained germanium [93] are currently heavily being investigated, these approaches are still in their infancy and considerable improvement is needed before efficient lasers can be demonstrated using these techniques. The most viable wafer scale type approach for realizing lasers on a silicon waveguide platform today is the hybrid integration platform whereby III-V epitaxial layers are integrated on silicon using wafer-to-wafer and die-to-wafer bonding techniques [12]. Following this bonding step the substrate of the III-V wafers is removed and the lasers or amplifiers are further processed using standard processing techniques, including mesa etching and metallization. Such hybrid III-V on silicon lasers have mostly been demonstrated starting from InP active layers and output powers above $20 \mathrm{~mW}$, threshold currents below $20 \mathrm{~mA}$, operation till $80{ }^{\circ} \mathrm{C}$ and a wavelength tuning range of more than $45 \mathrm{~nm}$ were demonstrated [29], [94]-[96]. Recently also GaAs and GaSb-based devices were demonstrated [97], [98]. In some cases the mode in the active section is a true hybrid mode, spread out over both the III-V and the silicon layers [99]. In

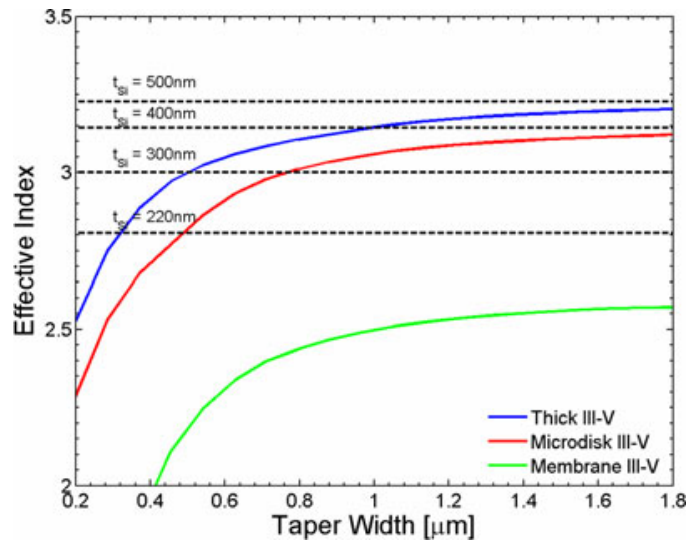

Fig. 14. Effective mode index of silicon slab waveguides with varying thickness (horizontal dashed black lines) and of three representative III-V wave guides, as a function of their width.

other cases, the mode in the active section is largely located in the III-V layers [95], [100]. In all cases the aim is to couple the mode fully to the silicon guide at the end of the active section, e.g., using adiabatic tapers [27], [100], [101], where they can be integrated with e.g., DBR-mirrors, ring resonators or AWG routers [101]-[103] to create more complex laser structures. Also integration with active devices such as modulators has been demonstrated [96], [104].

In practice most of this study was carried out on thicker silicon waveguides ( $400 \mathrm{~nm}$ and above). The reason can be understood by having a look at Fig. 14. The horizontal lines in this figure depict the effective index of a slab layer of silicon with thicknesses of respectively $220,300,400$, and $500 \mathrm{~nm}$. In principle this is the highest index a waveguide fabricated in this layer can reach. The solid curves on the other hand give the effective index of 3 representative III-V epitaxial stacks, as a function of their width. We first focus on the blue line, which represents the effective index of a waveguide fabricated in a $2 \mu \mathrm{m}$ thick epitaxial stack, containing a $300 \mathrm{~nm}$ thick active layer. Details of this layer structure can be found in [29]. All simulations were carried out for a wavelength of $1550 \mathrm{~nm}$. It is immediately obvious that coupling from this layer to the $220 \mathrm{~nm}$ thick silicon layer will be very difficult: to allow for adiabatic coupling the index of both waveguides has to cross. For the $220 \mathrm{~nm}$ silicon layer and the thick III-V waveguide this only happens when we decrease the width of the latter below $0.3 \mu \mathrm{m}$, which is far from trivial in practice. Even if we decrease the thickness of the III-V layer stack to e.g., $500 \mathrm{~nm}$ (red in Fig. 14), as was used for realizing microdisk lasers (see [105] for details of this stack), the waveguide still has to be tapered down to below $0.5 \mu \mathrm{m}$. Only when using thin membranes (green in Fig. 14, $200 \mathrm{~nm}$ thick InP membrane), coupling to the $220 \mathrm{~nm}$ layer stack becomes trivial. If the thickness of the silicon layer is increased to $400 \mathrm{~nm}$ the width of the III-V waveguide can be increased to $1.0 \mu \mathrm{m}$ to ensure mode crossing. Such a width seems rather straightforward to define, even using standard contact mask lithography. Fig. 14 does not tell the full story however. Contrary to lateral adiabatic tapers, where the distance between both guides can be gradually increased following the mode crossing point, in this 


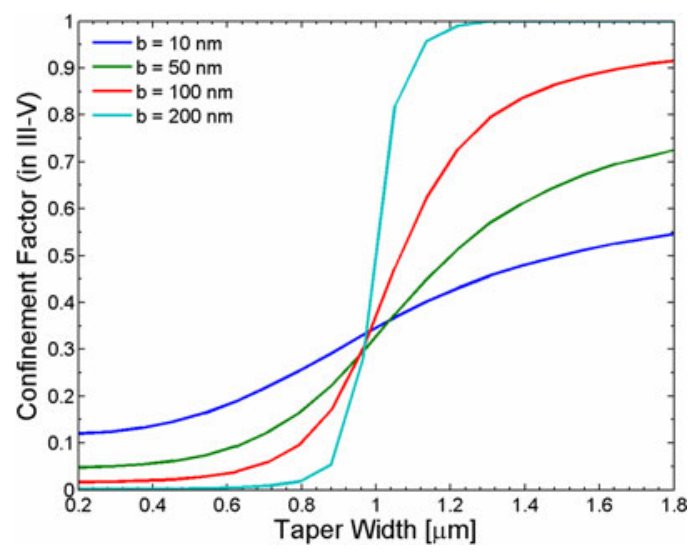

Fig. 15. Light confinement in the III-V layer as a function of the taper width for a III-V hybrid structure on top of a $2 \mu \mathrm{m}$ wide and $400 \mathrm{~nm}$ thick silicon waveguide, for different bonding layer thicknesses $b$.

vertical structure the distance between the center of both guides remains constant and relatively low. As a consequence, even when the mode has been pushed down to the silicon layer still a large fraction of the light remains in the III-V guide, which is problematic because it will result in losses and unwanted reflections at the end of the III-V guide. This is illustrated in Fig. 15, which shows the light confinement factor in the III-V layers as a function of the III-V taper width (assuming a $2 \mu \mathrm{m}$ wide and $400 \mathrm{~nm}$ thick silicon waveguide, and the $2 \mu \mathrm{m}$ thick III-V layer stack discussed above). For thin bonding layers the confinement factor changes very gradually as a function of the taper width and even for very narrow tapers, a considerable fraction of the light remains in the III-V layers (note that we assumed the $200 \mathrm{~nm}$ InP bottom cladding remained unetched). If the bonding layer increases however, the mode switches much more abruptly between each layers around the mode matching point, and the amount of light remaining in the III-V layer becomes negligible. Using thicker bonding layers however will require a longer taper to keep mode transformation losses low. Returning to Fig. 14, we see that the effective index of the $500 \mathrm{~nm}$ thick silicon layer is quasi similar to that of the wider III-V waveguides. In this case a truly hybrid mode can form.

Another aspect which is worth considering is the influence of the silicon layer thickness on the strength of a grating defined in this silicon layer, below a III-V active layer, e.g., when realizing a distributed feedback laser. Fig. 16 shows the coupling factor as a function of the etch depth for a hybrid III-V/silicon grating, using the thick III-V layer stack discussed above $(2 \mu \mathrm{m})$, a $20 \mathrm{~nm}$ bonding layer and for three different silicon layer thicknesses $(t)$. It is obvious that for the $220 \mathrm{~nm}$ silicon layer the coupling factor remains relatively low, while for the thicker layers the coupling factor can become relatively strong.

To overcome the challenges involved with coupling directly to standard $220 \mathrm{~nm}$ thick silicon waveguides using adiabatic tapers, several groups have investigated alternative schemes. In one approach a small fraction of the light is coupled out evanescently while the main part of the light remains in the cavity formed by the III-V structure. The best known example is the microdisk laser shown in Fig. 17(a) [106], whereby the III-V disk forms

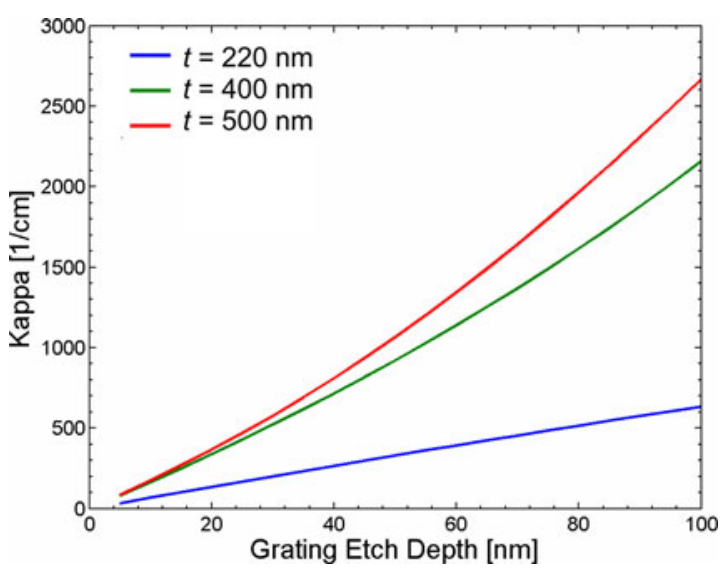

Fig. 16. Coupling factor for hybrid III-V silicon grating. (a)

(c)

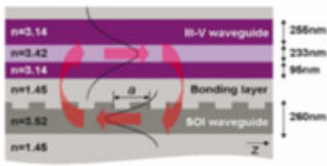

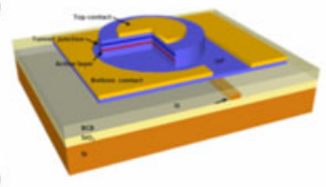

(b)

(d)

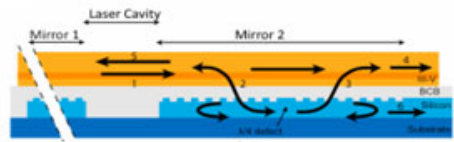

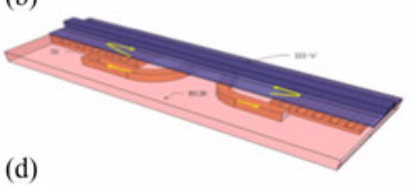

Fig. 17. (a) Evanescent coupling in microdisk laser (from [106]), (b) Evanescent intracavity coupling in DBR-laser (from [107], (c) Contradirectional coupling through grating in silicon layer (from [112]), and (d) Mirror formed by coupling to resonant cavity (from [113]).

the laser cavity and a few percent of the total circulating power is coupled out every roundtrip to an evanescently coupled silicon waveguide below the cavity. This structure has the additional advantage that the total III-V layer structure thickness can be reduced: since the whispering gallery mode is situated at the edge of the device it will not feel the metal top contact as long as the latter has a diameter somewhat smaller than the III-V disk diameter. Recently also an inline laser exploiting intracavity evanescent outcoupling was demonstrated [see Fig. 17(b)]. In this case the cavity is formed by two III-V/silicon hybrid DBR mirrors and light is coupled out evanescently through a directional coupler located in the center of the cavity [107]. Of course one can also decrease the thickness of the III-V layer to such an extent that direct coupling to the silicon layer becomes trivial, as for example has been done to demonstrate optically pumped amplifiers [108] and switches [109] or photonic crystal based microlasers [110]. The main challenge then is to make this stack compatible with electrical injection without introducing excessive losses through the top metal contact. In [111] an approach involving microstructuring of the upper InP-cladding was proposed to resolve this. However, this has yet to be demonstrated. An alternative approach to cope with the large mismatch between the III-V and silicon propagation constant is to include a grating in the design. In [112] contradirectional coupling through a corrugated silicon waveguide was used to couple light between these layer stacks [see Fig. 17(c)]. In [113], at each end side of the device light is coupled from 
the III-V stack to a cavity defined in the silicon layer stack [see Fig. 17(d)]. This approach has the advantage that the thickness of the layer stack can be increased compared to evanescently coupled devices.

From the above discussion it is obvious that realizing hybrid III-V on silicon lasers is more straightforward when using thicker silicon waveguide layers ( $400 \mathrm{~nm}$ and above) than when coupling to $220 \mathrm{~nm}$ or even $300 \mathrm{~nm}$ thick silicon. Unfortunately these thicker layer stacks are not available through the standard multi project wafer runs. Therefore it remains important to investigate novel approaches for realizing equally efficient lasers also on the standard silicon layer thicknesses.

\section{CONCLUSION}

Silicon photonics has reached an important crossroads. As the first commercial products are being deployed and many more are in development, we observe two important trends that are pushing the field towards greater standardization. The first is the need for the integration of a greater number of individual optical components on a chip, which means that whilst historically many components have been designed and optimized in isolation, they now have to be made compatible with a common integration platform. The second important trend is the move to a fabless design company model making use of existing CMOS foundries for fabrication-driven more by economical rather than technical concerns. For silicon photonics to be competitive within this model requires the production of large volumes of devices with a high fabrication yield, requirements which also demand a high degree of standardization for the integration platform and the fabrication processes used. The most basic standardization parameters are the layer thicknesses of the substrate, in particular that of the active silicon device layer. For the leading foundries that offer MPWs, a thickness of $220 \mathrm{~nm}$ has for now become the norm.

We have reviewed the silicon layer thickness considerations in the design of several individual components that are part of an integrated optical transceiver, namely passive components for waveguiding, wavelength selection, and light coupling as well as active components such as modulators and on-chip lasers produced by hybrid integration. A fairly complex picture arises from this review. For simple waveguides, standard $220 \mathrm{~nm}$ thick waveguides work well for TE but preclude the use of TM polarization, which is only well guided by waveguides with a thickness in excess of $250 \mathrm{~nm}$ when covered by a variety of cladding materials. This leads to sub-optimal performance of devices in particular with respect to propagation loss and back reflection. We have also discussed the implications for fabrication tolerances in wavelength selective devices and directional couplers which tend to be better for devices operating in TM polarization, considering in particular the improvements in silicon thickness uniformity achieved in $300 \mathrm{~mm}$ SOI wafers. Directionality of grating couplers is found to be optimized in silicon waveguides of approximately $350 \mathrm{~nm}$ thickness. For $220 \mathrm{~nm}$ silicon substrates, this problem is sometimes circumvented by the use of polycrystalline silicon overlayers in the grating region. For optical modulators we have defined a FOM for the design optimization. The results indicate an optimal silicon thickness of $360 \mathrm{~nm}$ and approximately $250 \mathrm{~nm}$ for TE and TM polarizations, respectively. Finally, we discussed the considerations for the hybrid integration of laser sources. Here we found that the strong coupling of laser and SOI waveguides necessary for good power transfer is achieved in a straightforward way only for thicker silicon waveguides of $400 \mathrm{~nm}$ and above, although more complex solutions for $220 \mathrm{~nm}$ thick waveguides are under investigation.

The review of the components then appears to indicate that a choice of $220 \mathrm{~nm}$ silicon is not ideal for any of the components we have discussed for the integration strategy of using monolithic silicon carrier-depletion modulators and hybrid lasers operating in the $1550 \mathrm{~nm}$ wavelength range. This conclusion may of course be expected for an integration platform that needs to make trade-offs for various components. More important to consider is the fact that in all cases we found that better performance or more robust fabrication could be achieved by using a thicker silicon waveguide layer, indicating that the sweet spot for integration may in fact be found for thicknesses greater than the now standard $220 \mathrm{~nm}$. Other more exploratory integration strategies, such as the use of graphene-based modulators overgrown on silicon, are beginning to be investigated. These strategies will certainly lead to different conclusions of the optimum silicon thickness. We believe that further research into the optimized silicon integration platform considering different options for the substrates will be of great importance in ultimately answering the question we have asked in the title and hope that this discussion paper has made a constructive contribution to this process.

\section{ACKNOWLEDGMENT}

The authors wish to acknowledge valuable discussions with Dr. Pavel Cheben of National Research Council Canada and Carlos Alonso-Ramos of University of Malaga in Spain.

\section{REFERENCES}

[1] D. A. Miller, "Rationale and challenges for optical interconnects to electronic chips," Proc. IEEE, vol. 88, no. 6, pp. 728-749, Jun. 2000.

[2] R. Soref, "The past, present, and future of silicon photonics," IEEE J. Sel. Topics Quantum Electron., vol. 12, no. 6, pp. 1678-1687, Nov./Dec. 2006.

[3] M. Asghari, "Silicon photonics: A low cost integration platform for datacom and telecom applications," presented at the Opt. Fiber Commun./Nat. Fiber Opt. Eng. Conf., San Diego, CA, USA, 2008.

[4] B. Jalali and S. Fathpour, "Silicon photonics," J. Lightw. Technol., vol. 24, no. 2, pp. 4600-4615, Dec. 2006.

[5] L. Kimerling, D. Ahn, A. Apsel, M. Beals, D. Carothers, Y.-K. Chen, T. Conway, D. Gill, M. Grove, and C.-Y. Hong, "Electronic-photonic integrated circuits on the CMOS platform," Proc. SPIE, vol. 6125, pp. 612502-1-612502-10, 2006.

[6] G. T. Reed, "Device physics: The optical age of silicon," Nature, vol. 427, pp. 595-596, 2004

[7] C. R. Doerr, P. J. Winzer, S. Chandrasekhar, M. Rasras, M. P. Earnshaw, J. S. Weiner, D. M. Gill, and Y.-K. Chen, "Monolithic silicon coherent receiver," presented at the Opt. Fiber Commun. Conf., San Diego, CA, USA, 2009

[8] A. V. Krishnamoorthy, R. Ho, X. Zheng, H. Schwetman, J. Lexau, P. Koka, G. Li, I. Shubin, and J. E. Cunningham, "Computer systems based on silicon photonic interconnects," Proc. IEEE, vol. 97, no. 7, pp. 1337-1361, Jul. 2009. 
[9] K. Okamoto, "Progress and technical challenge for planar waveguide devices: Silica and silicon waveguides," Laser Photon. Rev., vol. 6, pp. 14 23, 2012.

[10] D. J. Thomson, F. Y. Gardes, J.-M. Fedeli, S. Zlatanovic, Y. Hu, B. P. P. Kuo, E. Myslivets, N. Alic, S. Radic, and G. Z. Mashanovich, "50-Gb/s silicon optical modulator," IEEE Photon. Technol. Lett., vol. 24, no. 4, pp. 234-236, Feb. 2012

[11] G. T. Reed, G. Mashanovich, F. Gardes, and D. Thomson, "Silicon optical modulators," Nature Photon., vol. 4, pp. 518-526, 2010.

[12] G. Roelkens, L. Liu, D. Liang, R. Jones, A. Fang, B. Koch, and J. Bowers, "III-V/silicon photonics for on-chip and intra-chip optical interconnects," Laser Photon. Rev., vol. 4, pp. 751-779, 2010.

[13] Y. Painchaud, M. Pelletier, M. Poulin, F. Pelletier, C. Latrasse, G. Robidoux, S. Savard, J.-F. Gagne, V. Trudel, and M.-J. Picard, "Ultracompact coherent receiver based on hybrid integration on silicon," presented at the Opt. Fiber Commun. Conf. (Opt. Soc. Amer.), Anaheim, CA, USA, 2013

[14] Y. Urino, Y. Noguchi, M. Noguchi, M. Imai, M. Yamagishi, S. Saitou, N. Hirayama, M. Takahashi, H. Takahashi, and E. Saito, "Demonstration of 12.5-Gbps optical interconnects integrated with lasers, optical splitters, optical modulators and photodetectors on a single silicon substrate," presented at the Eur. Conf. Exhib. Opt. Commun. (Opt. Soc. Amer.), Amsterdam, The Netherlands, 2012.

[15] C. Kopp, S. Bernabe, B. B. Bakir, J. Fedeli, R. Orobtchouk, F. Schrank, H. Porte, L. Zimmermann, and T. Tekin, "Silicon photonic circuits: OnCMOS integration, fiber optical coupling, and packaging," IEEE J. Sel. Topics Quantum Electron., vol. 17, no. 3, pp. 498-509, May/Jun. 2011.

[16] Y. Urino, Y. Noguchi, M. Noguchi, M. Imai, M. Yamagishi, S. Saitou, N. Hirayama, M. Takahashi, H. Takahashi, E. Saito, M. Okano, T. Shimizu, N. Hatori, M. Ishizaka, T. Yamamoto, T. Baba, T. Akagawa, S. Akiyama, T. Usuki, D. Okamoto, M. Miura, J. Fujikata, D. Shimura, H. Okayama, H. Yaegashi, T. Tsuchizawa, K. Yamada, M. Mori, T. Horikawa, T. Nakamura, and Y. Arakawa, "Demonstration of 12.5Gbps optical interconnects integrated with lasers, optical splitters, optical modulators and photodetectors on a single silicon substrate," Opt. Exp., vol. 20, pp. B256-B263, 2012

[17] J. Niehusmann, A. Vorckel, P. H. Bolivar, T. Wahlbrink, W. Henschel, and H. Kurz, "Ultrahigh-quality-factor silicon-on-insulator microring resonator," Opt. Lett., vol. 29, pp. 2861-2863, 2004.

[18] D.-X. Xu, A. Densmore, A. Delâge, P. Waldron, R. McKinnon, S. Janz, J. Lapointe, G. Lopinski, T. Mischki, and E. Post, "Folded cavity SOI microring sensors for high sensitivity and real time measurement of biomolecular binding," Opt. Exp., vol. 16, pp. 15137-15148, 2008.

[19] W. Bogaerts, P. De Heyn, T. Van Vaerenbergh, K. De Vos, S. Kumar Selvaraja, T. Claes, P. Dumon, P. Bienstman, D. Van Thourhout, and R. Baets, "Silicon microring resonators," Laser Photon. Rev., vol. 6, pp. 47-73, 2012.

[20] Y. Ding, L. Liu, C. Peucheret, J. Xu, H. Ou, K. Yvind, X. Zhang, and D. Huang, "Towards polarization diversity on the SOI platform with simple fabrication process," IEEE Photon. Technol. Lett., vol. 23, no. 23, pp. 1808-1810, Dec. 2011.

[21] W. Bogaerts, D. Taillaert, P. Dumon, D. Van Thourhout, and R. Baets, "A polarization-diversity wavelength duplexer circuit in silicon-on-insulator photonic wires," Opt. Exp., vol. 15, pp. 1567-1578, 2007.

[22] P. Dong, L. Chen, and Y.-k. Chen, "High-speed low-voltage singledrive push-pull silicon Mach-Zehnder modulators," Opt. Exp., vol. 20, pp. 6163-6169, 2012.

[23] F. Gardes, A. Brimont, P. Sanchis, G. Rasigade, D. Marris-Morini, L. O'Faolain, F. Dong, J. Fedeli, P. Dumon, and L. Vivien, "High-speed modulation of a compact silicon ring resonator based on a reverse-biased pn diode," Opt. Exp., vol. 17, pp. 21986-21991, 2009.

[24] D. Marris-Morini, L. Vivien, J. M. Fedeli, E. Cassan, P. Lyan, and S. Laval, "Low loss and high speed silicon optical modulator based on a lateral carrier depletion structure," Opt. Exp., vol. 16, pp. 334-339, 2008.

[25] A. Biberman, S. Manipatruni, N. Ophir, L. Chen, M. Lipson, and K. Bergman, "First demonstration of long-haul transmission using silicon microring modulators," Opt. Exp., vol. 18, pp. 15544-15552, 2010.

[26] L. Vivien, M. Rouvière, J.-M. Fédéli, D. Marris-Morini, J. F. Damlencourt, J. Mangeney, P. Crozat, L. El Melhaoui, E. Cassan, and $\mathrm{X}$. Le Roux, "High speed and high responsivity germanium photodetector integrated in a silicon-on-insulator microwaveguide," Opt. Exp., vol. 15, pp. 9843-9848, 2007.

[27] B. Ben Bakir, A. Descos, N. Olivier, D. Bordel, P. Grosse, E. Augendre, L. Fulbert, and J. Fedeli, "Electrically driven hybrid Si/III-V Fabry-
Perot lasers based on adiabatic mode transformers," Opt. Exp., vol. 19, pp. 10317-10325, 2011.

[28] D. Liang and J. E. Bowers, "Recent progress in lasers on silicon," Nature Photon., vol. 4, pp. 511-517, 2010.

[29] S. Keyvaninia, G. Roelkens, D. Van Thourhout, C. Jany, M. Lamponi, A. Le Liepvre, F. Lelarge, D. Make, G.-H. Duan, and D. Bordel, "Demonstration of a heterogeneously integrated III-V/SOI single wavelength tunable laser," Opt. Exp., vol. 21, pp. 3784-3792, 2013.

[30] M. Hochberg and T. Baehr-Jones, "Towards fabless silicon photonics,' Nature Photon., vol. 4, pp. 492-494, 2010.

[31] P. Dumon, W. Bogaerts, R. Baets, J.-M. Fedeli, and L. Fulbert, "Towards foundry approach for silicon photonics: Silicon photonics platform ePIXfab," Electron. Lett., vol. 45, pp. 581-582, 2009.

[32] J. S. Orcutt, B. Moss, C. Sun, J. Leu, M. Georgas, J. Shainline, E. Zgraggen, H. Li, J. Sun, and M. Weaver, "Open foundry platform for high-performance electronic-photonic integration," Opt. Exp., vol. 20, pp. 12222-12232, 2012

[33] A. E.-J. Lim, J. Song, Q. Fang, C. Li, X. Tu, N. Duan, K. K. Chen, R. P. C. Tern, and T.-Y. Liow, "Review of silicon foundry efforts," IEEE J. Sel. Topics Quantum Electron., vol. 20, no. 4, Jul./Aug. 2014.

[34] A. Mekis, S. Abdalla, P. M. De Dobbelaere, D. Foltz, S. Gloeckner, S. Hovey, S. Jackson, Y. Liang, M. Mack, and G. Masini, "Scaling CMOS photonics transceivers beyond $100 \mathrm{~Gb} / \mathrm{s}$," in Proc. SPIE OPTO(Int. Soc. Opt. Photon.), 2012, pp. 82650A-82658A

[35] F. Boeuf, S. Crémer, N. Vulliet, T. Pinguet, A. Mekis, G. Masini, L. Verslegers, P. Sun, A. Ayazi, N.-K. Hon, S. Sahni, Y. Chi, B. Orlando, D. Ristoiu, A. Farcy, F. Leverd, L. Broussous, D. Pelissier-Tanon, C. Richard, L. Pinzelli, R. Beneyton, O. Gourhant, E. Gourvest, Y. LeFriec, D. Monnier, P. Brun, M. Guillermet, D. Benoit, K. Haxaire, J. R Manouvrier, S. Jan, H. Petiton, J. F. Carpentier, T. Quémerais, C. Durand, D. Gloria, M. Fourel, E. Batail, F. Baron, P. Delpech, L. Salager, P. De Dobbelaere, and B. Sautreuil, "A multi-wavelength 3D-compatible silicon photonics platform on $300 \mathrm{~mm}$ SOI wafers for 25 Gb/s applications," in Proc. Int. Electron. Devices Meeting, paper 13.3, 2013.

[36] A. V. Krishnamoorthy, X. Zheng, G. Li, J. Yao, T. Pinguet, A. Mekis, H. Thacker, I. Shubin, Y. Luo, and K. Raj, "Exploiting CMOS manufacturing to reduce tuning requirements for resonant optical devices," IEEE Photon. J., vol. 3, no. 3, pp. 567-579, Jun. 2011.

[37] A. Mekis, S. Gloeckner, G. Masini, A. Narasimha, T. Pinguet, S. Sahni, and P. De Dobbelaere, "A grating-coupler-enabled CMOS photonics platform," IEEE J. Select. Topics Quantum Electron., vol. 17, no. 3, pp. 597-608, May/Jun. 2011.

[38] L. Tsybeskov and D. J. Lockwood, "Silicon-germanium nanostructures for light emitters and on-chip optical interconnects," Proc. IEEE, vol. 97, no. 7, pp. 1284-1303, Jul. 2009.

[39] R. E. Camacho-Aguilera, Y. Cai, N. Patel, J. T. Bessette, M. Romagnoli, L. C. Kimerling, and J. Michel, "An electrically pumped germanium laser," Opt. Exp., vol. 20, pp. 11316-11320, 2011.

[40] E. R. Fuchs, R. E. Kirchain, and S. Liu, "The future of silicon photonics: Not so fast? insights from $100 \mathrm{G}$ ethernet LAN transceivers," J. Lightw. Technol., vol. 29, no. 15, pp. 2319-2326, Aug. 2011

[41] A. Narasimha, B. Analui, Y. Liang, T. J. Sleboda, S. Abdalla, E. Balmater, S. Gloeckner, D. Guckenberger, M. Harrison, and R. G. Koumans, "A fully integrated $4 \times 10-\mathrm{Gb} / \mathrm{s}$ DWDM optoelectronic transceiver implemented in a standard $0.13 \mu \mathrm{m}$ CMOS SOI technology," IEEE J. SolidState Circuits, vol. 42, no. 12, pp. 2736-2744, Dec. 2007.

[42] W. A. Zortman, D. C. Trotter, and M. R. Watts, "Silicon photonics manufacturing," Opt. Exp., vol. 18, pp. 23598-23607, 2010.

[43] M. A. Popovic, T. Barwicz, E. P. Ippen, and F. X. Kärtner, "Global design rules for silicon microphotonic waveguides: Sensitivity, polarization and resonance tunability," presented at the Conf. Lasers Electro-Opt. (Opt. Soc. Amer.), Long Beach, CA, USA, 2006.

[44] A. Prinzen, M. Waldow, and H. Kurz, "Fabrication tolerances of SOI based directional couplers and ring resonators," Opt. Exp., vol. 21, pp. 17212-17220, 2013

[45] L. Liu, Y. Ding, K. Yvind, and J. M. Hvam, "Efficient and compact TE-TM polarization converter built on silicon-on-insulator platform with a simple fabrication process," Opt. Lett., vol. 36, pp. 1059-1061, 2011.

[46] P. Dumon. (2007) "Ultra-compact integrated optical filters in silicon-on-insulator by means of wafer-scale technology, Ph.D. Thesis dissertation, Ghent University. [Online]. Available: http://photonics.intec.ugent.be/publications/PhD.asp?ID $=156$.

[47] Y. A. Vlasov and S. J. McNab, "Losses in single-mode silicon-oninsulator strip waveguides and bends," Opt. Exp., vol. 12, pp. 1622-1631, 2004. 
[48] V. R. Almeida, C. A. Barrios, R. R. Panepucci, and M. Lipson, "Alloptical control of light on a silicon chip," Nature, vol. 431, pp. 10811084, 2004.

[49] Y. Ding, L. Liu, C. Peucheret, and H. Ou, "Fabrication tolerant polarization splitter and rotator based on a tapered directional coupler," Opt. Exp., vol. 20, pp. 20021-20027, 2012.

[50] S. K. Selvaraja. (2011) "Wafer scale fabrication technology for silicon photonic integrated circuits," Ph.D. Thesis dissertation, Ghent University. [Online]. Available: http://photonics.intec.ugent.be/ download/phd_172.pdf.

[51] SOITEC, [Online]. Available: http://www.soitec.com/pdf/SoitecXtreme-SOI-product-brief.pdf

[52] S. K. Selvaraja, W. Bogaerts, P. Dumon, D. Van Thourhout, and R. Baets, "Subnanometer linewidth uniformity in silicon nanophotonic waveguide devices using CMOS fabrication technology," IEEE J. Sel. Topics Quantum Electron., vol. 16, no. 1, pp. 316-324, Jan./Feb. 2009.

[53] IMEC internal results (unpublished).

[54] Y. Tanushi, T. Kita, M. Toyama, M. Seki, K. Koshino, N. Yokoyama, M. Ohtsuka, A. Sugiyama, E. Ishitsuka, T. Sano, T. Horikawa, and H. Yamada, "Uniform characteristics of Si-wire waveguide devices fabricated on $300 \mathrm{~mm}$ SOI wafers by using ArF immersion lithography," in Proc. IEEE 10th Int. Conf. Group IV Photon., 2013, pp. 105-106.

[55] G. Li, J. Yao, H. Thacker, A. Mekis, X. Zheng, I. Shubin, Y. Luo, J.-h. Lee, K. Raj, and J. E. Cunningham, "Ultralow-loss, high-density SOI optical waveguide routing for macrochip interconnects," Opt. Exp., vol. 20, pp. 12035-12039, 2012.

[56] W. Bogaerts and S. Selvaraja, "Compact single-mode silicon hybrid rib/strip waveguide with adiabatic bends," IEEE Photon. J., vol. 3, no. 3, pp. 422-432, Jun. 2011.

[57] S. Selvaraja, L. Fernandez, M. Vanslembrouck, J.-L. Everaert, P. Dumon, J. Van Campenhout, W. Bogaerts, and P. Absil, "Si photonic device uniformity improvement using wafer-scale location specific processing," in Proc. IEEE Photon. Conf., 2012, pp. 725-726.

[58] M. Webster, R. Pafchek, A. Mitchell, and T. Koch, "Width dependence of inherent TM-mode lateral leakage loss in silicon-on-insulator ridge waveguides," IEEE Photon. Technol. Lett., vol. 19, no. 6, pp. 429-431, Mar. 2007.

[59] S. Xiao, M. H. Khan, H. Shen, and M. Qi, "Compact silicon microring resonators with ultra-low propagation loss in the C band," Opt. Exp., vol. 15, pp. 14467-14475, 2007.

[60] F. Morichetti, A. Canciamilla, C. Ferrari, M. Torregiani, A. Melloni, and M. Martinelli, "Roughness induced backscattering in optical silicon waveguides," Phys. Rev. Lett., vol. 104, pp. 33902-1-033902-4, 2010.

[61] Z. Sheng, C. Qiu, H. Li, L. Li, A. Pang, A. Wu, X. Wang, S. Zou, and F. Gan, "Low loss silicon nanowire waveguide fabricated with $0.13 \mathrm{um}$ CMOS technology," presented at the Asia Commun. Photon. Conf. (Opt. Soc. Amer.), Guangzhou, China, 2012.

[62] S. K. Selvaraja, G. Murdoch, A. Milenin, C. Delvaux, S. P. Patrick Ong, G. S. D. Vermeulen, G. Winroth, P. Verheyen, G. Lepage, R. Baets, W. Bogaerts, J. Van Campenhout, and P. Absil, "Advanced 300-mm waferscale patterning for silicon photonics devices with record low loss and phase errors," in Proc. 17th OptoElectron. Commun. Conf., 2012, pp. 15-16.

[63] F. Morichetti, A. Canciamilla, M. Martinelli, A. Samarelli, R. De La Rue, M. Sorel, and A. Melloni, "Coherent backscattering in optical microring resonators," Appl. Phys. Lett., vol. 96, pp. 081112-1-081112-3, 2010.

[64] P. De Heyn, D. Vermeulen, D. Van Thourhout, and G. Roelkens, "Siliconon-insulator all-pass microring resonator using a polarization rotating coupling section," IEEE Photon. Technol. Lett., vol. 24, no. 14, pp. 11761178, Jul. 2012.

[65] K. Kakihara, N. Kono, K. Saitoh, and M. Koshiba, "Full-vectorial finite element method in a cylindrical coordinate system for loss analysis of photonic wire bends," Opt. Exp., vol. 14, pp. 11128-11141, 2006.

[66] A. M. Prabhu, A. Tsay, Z. Han, and V. Van, "Extreme miniaturization of silicon add-drop microring filters for VLSI photonics applications," IEEE Photon. J., vol. 2, no. 3, pp. 436-444, Jun. 2010.

[67] Z. Sheng, D. Dai, and S. He, "Comparative study of losses in ultrasharp silicon-on-insulator nanowire bends," IEEE J. Select. Topics Quantum Electron., vol. 15, no. 5, pp. 1406-1412, Sep./Oct. 2009.

[68] S. Xiao, M. H. Khan, H. Shen, and M. Qi, "Modeling and measurement of losses in silicon-on-insulator resonators and bends," Opt. Exp., vol. 15, pp. 10553-10561, 2007.

[69] D.-X. Xu, A. Densmore, A. Delâge, P. Waldron, R. McKinnon, S. Janz, J. Lapointe, G. Lopinski, T. Mischki, E. Post, P. Cheben, and J. H. Schmid, "Folded cavity SOI microring sensors for high sensitivity and real time measurement of biomolecular binding," Opt. Exp., vol. 16, pp. 15137-15148, 2008
[70] D.-X. Xu, A. Densmore, J. Schmid, R. Ma, M. Vachon, S. Janz, R. McKinnon, J. Lapointe, A. Delâge, and E. Post, "Spiral cavity Si wire resonators as label-free biosensors," presented at the Integr. Photon. Nanophoton. Res. Appl., Honolulu, HI, USA, 2009.

[71] W. R. McKinnon, D. X. Xu, C. Storey, E. Post, A. Densmore, A. Delâge, P. Waldron, J. H. Schmid, and S. Janz, "Extracting coupling and loss coefficients from a ring resonator," Opt. Exp., vol. 17, pp. 18971-18982, 2009.

[72] R. Soref, "Mid-infrared photonics in silicon and germanium," Nature Photon., vol. 4, pp. 495-497, 2010.

[73] E. D. Palik, Handbook of Optical Constants of Solids: Index. Amsterdam, The Netherlands: Elsevier, 1998.

[74] X. Liu, B. Kuyken, G. Roelkens, R. Baets, R. M. Osgood Jr, and W. M. Green, "Bridging the mid-infrared-to-telecom gap with silicon nanophotonic spectral translation," Nature Photon., vol. 6, pp. 667-671, 2012.

[75] Z. Cheng, X. Chen, C. Wong, K. Xu, C. K. Fung, Y. Chen, and H. K. Tsang, "Focusing subwavelength grating coupler for mid-infrared suspended membrane waveguide," Opt. Lett., vol. 37, pp. 1217-1219, 2012.

[76] M. M. Milosevic, M. Nedeljkovic, T. M. Ben Masaud, E. Jaberansary, H. M. Chong, N. G. Emerson, G. T. Reed, and G. Z. Mashanovich, "Silicon waveguides and devices for the mid-infrared," Appl. Phys. Lett. vol. 101, pp. 121105-1-121105-4, 2012.

[77] M. Nedeljkovic, A. Khokhar, Y. Hu, X. Chen, J. S. Penades, S. Stankovic, H. Chong, D. Thomson, F. Gardes, and G. Reed, "Silicon photonic devices and platforms for the mid-infrared," Opt. Mater. Exp., vol. 3, pp. 1205-1214, 2013.

[78] A. Delage, D. X. Xu, R. W. McKinnon, E. Post, P. Waldron, J. Lapointe, C. Storey, A. Densmore, S. Janz, and B. Lamontagne, "Wavelengthdependent model of a ring resonator sensor excited by a directional coupler," J. Lightw. Technol., vol. 27, no. 9, pp. 1172-1180, May 2009.

[79] F. Xia, L. Sekaric, and Y. A. Vlasov, "Mode conversion losses in silicon-on-insulator photonic wire based racetrack resonators," Opt. Exp., vol. 14, pp. 3872-3886, 2006.

[80] C.-W. Tseng, C.-W. Tsai, K.-C. Lin, M.-C. Lee, and Y.-J. Chen, "Study of coupling loss on strongly-coupled, ultra compact microring resonators," Opt. Exp., vol. 21, pp. 7250-7257, 2013.

[81] R. Halir, P. Cheben, J. Schmid, R. Ma, D. Bedard, S. Janz, D.-X. Xu, A. Densmore, J. Lapointe, and I. Molina-Fernandez, "Continuously apodized fiber-to-chip surface grating coupler with refractive index engineered subwavelength structure," Opt. Lett., vol. 35, pp. 3243-3245, 2010 .

[82] X. Chen, C. Li, C. K. Fung, S. M. Lo, and H. K. Tsang, “Apodized waveguide grating couplers for efficient coupling to optical fibers," IEEE Photon. Technol. Lett., vol. 22, no. 15, pp. 1156-1158, Aug. 2010.

[83] R. Halir, P. Cheben, S. Janz, D.-X. Xu, I. Molina-Fernandez, and J. G. Wangüemert-Perez, "Waveguide grating coupler with subwavelength microstructures," Opt. Lett., vol. 34, pp. 1408-1410, 2009.

[84] F. Van Laere, G. Roelkens, M. Ayre, J. Schrauwen, D. Taillaert, D. Van Thourhout, T. F. Krauss, and R. Baets, "Compact and highly efficient grating couplers between optical fiber and nanophotonic waveguides," $J$. Lightw. Technol., vol. 25, no. 1, pp. 151-156, Jan. 2007.

[85] C. Alonso-Ramos, A. Ortega-Monux, L. Zavargo-Peche, R. Halir, J. de Oliva-Rubio, I. Molina-Fernandez, P. Cheben, D.-X. Xu, S. Janz, and N. Kim, "Single-etch grating coupler for micrometric silicon rib waveguides," Opt. Lett., vol. 36, pp. 2647-2649, 2011.

[86] G. Roelkens, D. Van Thourhout, and R. Baets, "High efficiency siliconon-insulator grating coupler based on a poly-silicon overlay," Opt. Exp., vol. 14, pp. 11622-11630, 2006.

[87] F. Gardes, G. Reed, N. Emerson, and C. Png, "A sub-micron depletiontype photonic modulator in silicon on insulator," Opt. Exp., vol. 13, pp. 8845-8854, 2005.

[88] M. R. Watts, W. A. Zortman, D. C. Trotter, R. W. Young, and A. L. Lentine, "Low-voltage, compact, depletion-mode, silicon MachZehnder modulator," IEEE J. Sel. Topics Quantum Electron., vol. 16, no. 1, pp. 159-164, Jan./Feb. 2010.

[89] Z.-Y. Li, D.-X. Xu, W. R. McKinnon, S. Janz, J. H. Schmid, P. Cheben, and J.-Z. Yu, "Silicon waveguide modulator based on carrier depletion in periodically interleaved PN junctions," Opt. Exp., vol. 17, pp. 1594715958, 2009.

[90] H. Xu, X. Xiao, X. Li, Y. Hu, Z. Li, T. Chu, Y. Yu, and J. Yu, "High speed silicon Mach-Zehnder modulator based on interleaved PN junctions," Opt. Exp., vol. 20, pp. 15093-15099, 2012.

[91] D. Marris-Morini, C. Baudot, J. Fedeli, G. Rasigade, N. Vulliet, A. Souhaite, M. Ziebell, P. Rivallin, S. Olivier, and P. Crozat, "Low 
loss $40 \mathrm{Gbit} / \mathrm{s}$ silicon modulator based on interleaved junctions and fabricated on $300 \mathrm{~mm}$ SOI wafers," Opt. Exp., vol. 21, pp. 22471-22475, 2013.

[92] A. D. Lee and A. J. Seeds, "InAs/GaAs quantum-dot lasers monolithically grown on $\mathrm{Si}, \mathrm{Ge}$, and Ge-on-Si substrates," IEEE J. Select. Topics Quantum Electron., vol. 19, no. 4, 1901107, 2013.

[93] X. Sun, J. Liu, L. C. Kimerling, and J. Michel, "Toward a germanium laser for integrated silicon photonics," IEEE J. Select. Topics Quantum Electron., vol. 16, no. 1, pp. 124-131, Jan./Feb. 2010.

[94] B. Koch, E. J. Norberg, B. Kim, J. Hutchison, J. H. Shin, G. Fish, and A. Fang, "Integrated silicon photonic laser sources for telecom and datacom," presented at the Opt. Fiber Commun. Conf. Expo., Anaheim, CA, USA, 2013.

[95] A. Descos, C. Jany, D. Bordel, H. Duprez, G. B. D. Farias, P. Brianceau, S. Menezo, and B. B. Bakir, "Heterogeneously integrated III-V/Si distributed Bragg reflector laser with adiabatic coupling," presented at the Eur. Conf. Opt. Commun., London, U.K., 2013.

[96] G.-H. Duan, C. Jany, A. Le Liepvre, M. Lamponi, A. Accard, F. Poingt, D. Make, F. Lelarge, S. Messaoudene, and D. Bordel, "Integrated hybrid III-V/Si laser and transmitter," in Proc. Int. Conf. Indium Phosphide Related Mater., Santa Barbara, CA, USA, 2012, pp. 16-19.

[97] N. Hattasan, A. Gassenq, L. Cerutti, J. B. Rodriguez, E. Tournie, and G. Roelkens, "Integrated thin-film GaSb-based Fabry-Perot lasers: Towards a fully integrated spectrometer on a SOI waveguide circuit," in Proc. SPIE OPTO (Int. Soc. Opt. Photon.), 2013, pp. 863114-863118.

[98] K. Tanabe, K. Watanabe, and Y. Arakawa, "1.3 um InAs/GaAs quantum dot lasers on Si rib structures with current injection across direct-bonded GaAs/Si heterointerfaces," Opt. Exp., vol. 20, pp. B315-B321, 2012.

[99] A. W. Fang, H. Park, R. Jones, O. Cohen, M. J. Paniccia, and J. E. Bowers, "A continuous-wave hybrid AlGaInAs-silicon evanescent laser," IEEE Photon. Technol. Lett., vol. 18, no. 10, pp. 1143-1145, May 2006.

[100] M. Lamponi, S. Keyvaninia, C. Jany, F. Poingt, F. Lelarge, G. D. Valicourt, G. Roelkens, D. V. Thourhout, S. Messaoudene, J. Fedeli, G. H. Duan, and S. Member, "Low-threshold heterogeneously integrated InP / SOI lasers with a double adiabatic taper coupler," IEEE Photon. Technol. Lett., vol. 24, no. 1, pp. 76-78, Jan. 2012.

[101] G. Kurczveil, P. Pintus, M. J. R. Heck, J. D. Peters, and J. E. Bowers, "Characterization of insertion loss and back reflection in passive hybrid silicon tapers," IEEE Photon. J., vol. 5, no. 2, 6600410, Apr. 2013.

[102] S. Keyvaninia, S. Verstuyft, S. Pathak, F. Lelarge, G.-H. Duan, D. Bordel, J.-M. Fedeli, T. de Vries, E. Smalbrugge, E.-J. Geluk, J. Bolk, G. Roelkens, and D. Van Thourhout, "III-V-on-silicon multi-frequency lasers," Opt. Exp., vol. 21, pp. 13675-13683, 2013.

[103] A. L. Liepvre, A. Accard, F. Poingt, C. Jany, M. Lamponi, D. Make, F. Lelarge, J.-M. Fedeli, S. Messaoudene, D. Bordel, and G.-H. Duan, "Wavelength selectable hybrid III-V/Si laser fabricated by wafer bonding," IEEE Photon. Technol. Lett., vol. 25, no. 16, pp. 1582-1585, Aug. 2013.

[104] B. Koch, A. Alduino, L. Liao, R. Jones, M. Morse, B. Kim, W.-Z. Lo, J. Basak, H.-F. Liu, H. Rong, M. Sysak, C. Krause, A. Liu, K. Sullivan, O. Dosunmu, N. Na, T. Yin, F. Haubensack, I. w. Hsieh, J. Heck, R. Beatty, J. Bovington, and M. Paniccia, "A $4 \times 12.5 \mathrm{~Gb} / \mathrm{s}$ CWDM Si photonics link using integrated hybrid silicon lasers," presented at the IEEE Conf. Lasers Electro-Opt., Baltimore, MD, USA, 2011.

[105] D. Van Thourhout, T. Spuesens, S. Kumar, S. Member, L. Liu, R. Kumar, G. Morthier, S. Member, P. Rojo-romeo, F. Mandorlo, P. Regreny, O. Raz, C. Kopp, and L. Grenouillet, "Nanophotonic devices for optical interconnect," IEEE J. Sel. Topics Quantum Electron., vol. 16, no. 5, pp. 1363 1375, Sep./Oct. 2010.

[106] J. Van Campenhout, P. Rojo-Romeo, P. Regreny, C. Seassal, D. Van Thourhout, S. Verstuyft, L. Di Cioccio, J. M. Fedeli, C. Lagahe, and R. Baets, "Electrically pumped InP-based microdisk lasers integrated with a nanophotonic silicon-on-insulator waveguide circuit," Opt. Exp., vol. 15, pp. 6744-6749, 2007.

[107] S. Keyvaninia, D. Van Thourhout, R. Baets, G. Roelkens, G.-H. Duan, F. Lelarge, J.-M. Fedeli, and S. Messaoudene, "Demonstration of a novel III-V-on-Si distributed feedback laser," presented at the Opt. Fiber Commun. Conf./Nat. Fiber Opt. Eng. Conf., Anaheim, CA, USA, 2013, Paper OTh1D.6.

[108] M. Tassaert, S. Keyvaninia, D. Van Thourhout, W. M. J. Green, Y. Vlasov, and G. Roelkens, "An optically pumped nanophotonic InP/InGaAlAs optical amplifier integrated on a SOI waveguide circuit," Opt. Quantum Electron., vol. 44, pp. 513-519, 2013.
[109] M. Tassaert, G. Roelkens, H. J. S. Dorren, D. Van Thourhout, and O. Raz, "Bias-free, low power and optically driven membrane InP switch on SOI for remotely configurable photonic packet switches," Opt. Exp., vol. 19, pp. 817-824, 2011.

[110] Y. Halioua, A. Bazin, P. Monnier, T. J. Karle, G. Roelkens, I. Sagnes, R. Raj, and F. Raineri, "Hybrid III-V semiconductor/silicon nanolaser," Opt. Exp., vol. 19, pp. 9221-9231, 2011.

[111] M. Tassaert, D. Van Thourhout, and G. Roelkens, "Strategies to increase the modal gain in heterogeneously integrated III-V amplifiers on siliconon-insulator," Opt. Quantum Electron., vol. 44, pp. 683-689, 2012.

[112] T. Dupont, L. Grenouillet, A. Chelnokov, and P. Viktorovitch, "Contradirectional coupling between III-V stacks and silicon-on-insulator corrugated waveguides for laser emission by distributed feedback effect," IEEE Photon. Technol. Lett., vol. 22, no. 19, pp. 1413-1415, Oct. 2010.

[113] Y. de Koninck, F. Raineri, A. Bazin, R. Raj, G. Roelkens, and R. Baets, "Experimental demonstration of a hybrid III-V-on-silicon microlaser based on resonant grating cavity mirrors," Opt. Lett., vol. 38, pp. 2496 2498,2013

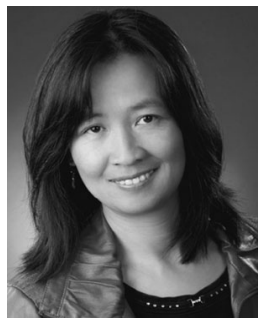

Dan-Xia Xu received the Ph.D. degree from Linköping University, Linköping, Sweden, in 1991, working on silicon-germanium HBTs and multiquantum-well tunneling diodes. She is currently a Senior Research Officer with National Research Council (NRC) Canada, Ottawa, ON, Canada, and an Adjunct Professor with Carleton University, Ottawa, ON, Canada. Since joining NRC, she has developed high-speed SiGe HBTs, SiGe, and silicide photodetectors, and pioneered the use of nickel silicide for deep submicron VLSI. She later switched her research field to integrated optics. In 2001-2002, she was part of the research team at Optenia Inc. that successfully developed the first commercial glass waveguide echelle grating demultiplexer. In 2003, she pioneered the study of cladding stress induced birefringence in SOI waveguides and its application for polarization independent operation in photonic components. In 2005, she initiated the work on SOI photonic wire biosensors. The NRC sensor team was the first worldwide to develop an evanescent field waveguide biosensor on the SOI platform, and holds the patent for this technology. Her current research interest is silicon photonics for optical communications and biological sensing. She has authored or coauthored more than 300 journals and peer-reviewed conference, several book chapters, and holds seven patents.

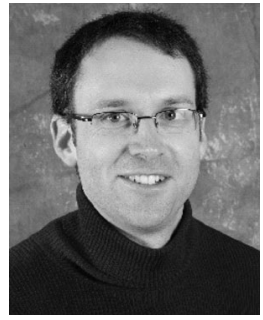

Jens H. Schmid received the Ph.D. degree from the University of British Columbia, Vancouver, BC, Canada, in 2004, for his work on in situ etching and molecular beam epitaxial regrowth on III-V semiconductors. He is a currently a Research Officer with the Information and Communications Technologies portfolio of the National Research Council (NRC) Canada and also an Adjunct Professor with the Department of Electronics, Carleton University, Ottawa, ON, Canada. After working for a year as a Research Scientist for VSM MedTech, a medical device company in Coquitlam, BC, where he developed fabrication processes for superconducting quantum interference devices; he joined the nanofabrication group at NRC in the fall of 2005 . His current research interests include the fabrication, design, characterization, and simulation of silicon photonic devices and nanostructures 


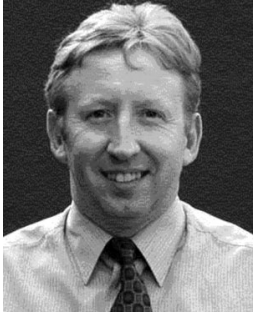

Graham T. Reed is a Professor of Silicon Photonics and Group Leader. He has recently joined the University of Southampton from the University of Surrey, where he was Professor of Optoelectronics, and was Head of the Department of Electronic Engineering from 2006 to 2012. He is a pioneer in the field of Silicon Photonics, and acknowledged as the individual who initiated the research field in the UK. He established the Silicon Photonics Research Group at Surrey in 1989. The first Silicon Photonics company in the world, Bookham Technology Inc., was founded by Reed's Ph.D. student, Dr. Andrew Rickman, and adopted the research developed in the Group. The Silicon Photonics Group have provided a series of world leading results since it's inception, and are particularly well known for their work on silicon optical modulators. He is a regular invited and contributing author to the major Silicon Photonics conferences around the world. He has served on numerous international conference committees, and has also chaired many others. To name but two, he has been co-chair, of the Silicon Photonics symposium at Photonics West since it was first established in 2006, and in 2011, he was co-chair of the prestigious Silicon Photonics conference, IEEE Group IV Photonics, held at the Royal Society in London. He is currently a member of five international conference committees, and has published more than 250 papers in the field of Silicon Photonics.

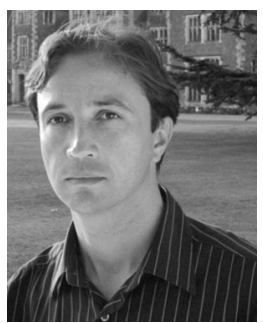

Goran Z. Mashanovich received the Dipl. Ing. and M.Sc. degrees in optoelectronics from the Faculty of Electrical Engineering, University of Belgrade, Belgrade, Serbia, and the Ph.D. degree in silicon photonics from the University of Surrey, Surrey, U.K. $\mathrm{He}$ is currently a Royal Society Research Fellow and Reader in silicon photonics at the Optoelectronics Research Centre, University of Southampton, Southampton, U.K. He has been a member of the Southampton Silicon Photonics Group since 2000, and is actively researching near- and mid-infrared photonic devices in group IV materials. He has published 160 papers in the field and is a member of several international conference committees related to photonics.

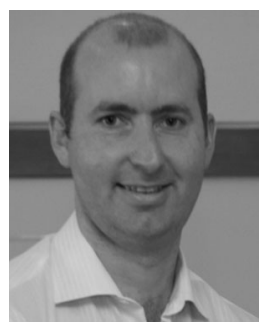

David J. Thomson is a Senior Research Fellow in the Optoelectronics Research Centre, University of Southampton. He started his silicon photonics research in 2004 as a Ph.D. student at the University of Surrey under the guidance of Prof. Graham Reed. His Ph.D. project involved investigating silicon based total internal reflection optical switches and more specifically methods of restricting free carrier diffusion within such devices. His research interests include optical modulation, optical switching, integration and packaging in silicon. In 2008, he took up a role as a Research Fellow in the same research group leading the work package on silicon optical modulators within the largest European silicon photonics project named HELIOS. Within this project, he designed the first silicon optical modulator operating at $50 \mathrm{Gbit} / \mathrm{s}$. He has presented invited talks at SPIE Photonics West, IEEE Group IV Photonics, and IEEE Photonics Global conferences, and in 2012 was selected to present his work at the SET for Britain event in the Houses of Parliament.

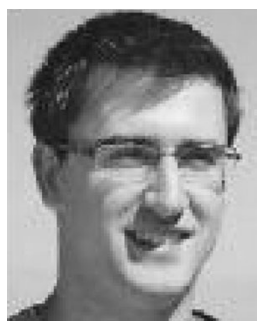

Milos Nedeljkovic received the M.Eng. degree in communications engineering from the University of Durham, Durham, U.K., in 2009. He is currently working toward the Ph.D. degree at the University of Southampton, Southampton, U.K. His research interests include photonic components and modulation mechanisms in Group-IV materials for mid-infrared wavelengths.

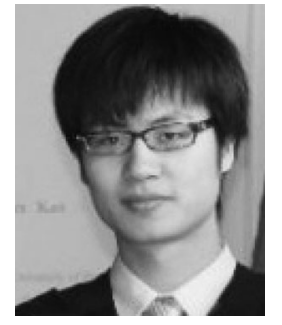

Xia Chen received the B.Eng. and Ph.D. degrees in electronics engineering from the Chinese University of Hong Kong, Hong Kong, in 2006 and 2010, respectively. He is currently a Royal Society Newton Fellow at Optoelectronics Research Centre, University of Southampton, Southampton, working on silicon photonics. He has authored and coauthored more than 50 papers in technical journals and international conferences. Dr. Chen received the TSMC Outstanding Student Research Award in 2010.

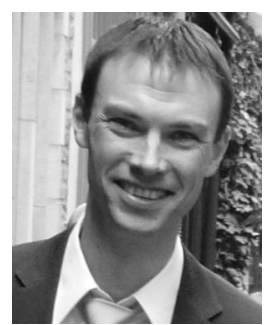

Dries Van Thourhout received the degree in physical engineering and the Ph.D. degree from Ghent University, Ghent, Belgium, in 1995 and 2000, respectively. From 2000 to 2002, he was with Lucent Technologies, Bell Laboratories, Crawford Hill, NJ, USA, working on InPInGaAsP monolithically integrated devices. In 2002, he joined the Department of Information Technology, Ghent University, where he is currently a Full Professor. His main interests include heterogeneous integration by wafer bonding for silicon photonics, intrachip optical interconnect, and WDM-devices. He holds an ERC-starting grant and is (co)author of more than 160 journal publications.

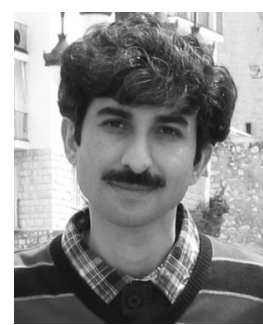

Shahram Keyvaninia received the degree in applied physics and the M.Sc. degree in photonics from the Sharif University of Technology, Tehran, Iran, and Institute for Color Science and Technology, Tehran. $\mathrm{He}$ has been working toward the Ph.D. degree in the Photonic Research Group, Interuniversity Microelectronics Center/Ghent University, Ghent, Belgium, since 2008. He developed the technology for integrating III-V material on top of silicon-on-insulator based on die-to-wafer bonding and new fabrication processes for photonic devices. His current research interests include the design, fabrication, and characterization of integrated photonic devices and heterogeneous integration of InP-on-silicon. Mr. Keyvaninia is a student member of the IEEE Laser and Electrooptics Society and Optical Society of America.

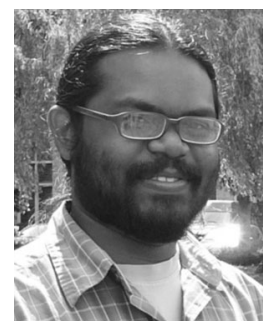

Shankar K. Selvaraja received the M.E. degree in optical communication from College of engineering, Anna University, Chennai, India, in 2004, and the M.Sc. degree in microsystems and microelectronics from University of Twente, Enschede, The Netherlands, in 2005. He received the Ph.D. degree for his work on wafer-scale fabrication technology for silicon photonic integrated circuits, in 2011. Since then he has been a Postdoctoral Researcher and currently a Silicon Photonics Integration Engineer at IMEC, Leuven, Belgium. His research interests include CMOS compatible device, process development and integration for onchip optical interconnects. He has authored or coauthored more than 90 journals and peer-reviewed conference articles. 